\title{
Mismatch repair deficiency is not sufficient to elicit tumor immunogenicity
}

Tyler Jacks ( $\square$ tjacks@mit.edu )

Massachusetts Institute of Technology https://orcid.org/0000-0001-5785-8911

\section{Peter Westcott}

MIT https://orcid.org/0000-0001-9436-4857

\section{Francesc Muyas}

EMBL-EBI

\section{Olivia Smith}

Massachusetts Institute of Technology https://orcid.org/0000-0002-2788-9917

\section{Haley Hauck}

Massachusetts Institute of Technology

\section{Nathan Sacks}

Massachusetts Institute of Technology

\section{Zackery Ely}

Koch Institute for Integrative Cancer Research at MIT

\section{Alex Jaeger}

Massachusetts Institute of Technology

\section{William Rideout III}

Koch Institute for Integrative Cancer Research at MIT

\section{Arjun Bhutkar}

MIT

\section{Daniel Zhang}

Massachusetts Institute of Technology

\section{Mary Beytagh}

Massachusetts Institute of Technology https://orcid.org/0000-0002-1882-1515

\section{David Canner}

Massachusetts Institute of Technology

\section{Roderick Bronson}

Dana Farber Cancer Center, Harvard Cancer Center

\section{Santiago Naranjo}

Massachusetts Institute of Technology

\section{Abbey Jin}

Massachusetts Institute of Technology

\section{J Patten}


Boston University https://orcid.org/0000-0002-9770-7525

Amanda Cruz

Massachusetts Institute of Technology

Isidro Cortes-Ciriano

European Molecular Biology Laboratory https://orcid.org/0000-0002-2036-494X

\section{Biological Sciences - Article}

Keywords: MMRd, tumor mutational burden, immune checkpoint blockade, CRISPR

Posted Date: September 10th, 2021

DOl: https://doi.org/10.21203/rs.3.rs-879464/v1

License: (a) This work is licensed under a Creative Commons Attribution 4.0 International License. Read Full License 
Mismatch repair deficiency is not sufficient to elicit tumor immunogenicity

Peter M K Westcott ${ }^{1}$, Francesc Muyas², Olivia Smith¹, Haley Hauck ${ }^{1}$, Nathan J Sacks ${ }^{1}$, Zackery A Ely ${ }^{1}$, Alex M Jaeger ${ }^{1}$, William M Rideout III ${ }^{1}$, Arjun Bhutkar ${ }^{1}$, Daniel Zhang ${ }^{1}$, Mary C Beytagh ${ }^{1}$, David A Canner ${ }^{1}$,

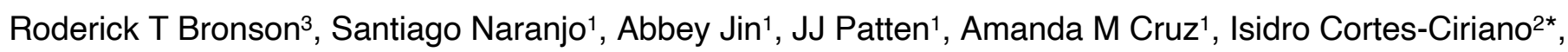
Tyler Jacks ${ }^{1,4^{*}}$

1David H. Koch Institute for Integrative Cancer Research, Massachusetts Institute of Technology, Cambridge, MA 02139, USA

European Molecular Biology Laboratory, European Bioinformatics Institute, Hinxton, Cambridge CB10 1SD, UK

${ }^{3}$ Rodent Histopathology Core, Harvard Medical School, Boston, MA 02115, USA

${ }^{4}$ Department of Biology, Massachusetts Institute of Technology, Cambridge, MA 02139, USA

*Corresponding authors, tjacks@mit.edu, icortes@ebi.ac.uk

\section{Summary}

DNA mismatch repair deficiency (MMRd) is associated with high tumor mutational burden (TMB), increased T cell infiltration, and remarkable responsiveness to immune checkpoint blockade (ICB) therapy ${ }^{1}$. Nevertheless, about half of MMRd tumors do not respond to ICB for unclear reasons. While cell line transplant models of MMRd have reinforced the importance of TMB in immune response $^{2,3}$, critical questions remain regarding the role of immunosurveillance in the evolution of MMRd tumors induced in vivo. Here, we developed autochthonous mouse models of lung and colon cancer with ablation of MMR via in vivo CRISPR/Cas9 targeting. Surprisingly, MMRd in these models did not increase T cell infiltration or response to ICB. Mechanistically, we showed this lack of immunogenicity to be driven by profound intratumoral heterogeneity. Studies in immune deficient animals further demonstrated that immunosurveillance in MMRd tumors has no impact 
on TMB but shapes the clonal architecture of neoantigens by exacerbating heterogeneity. These results provide important context for understanding immune evasion in cancers with high TMB and have major implications for therapies aimed at increasing TMB.

\section{Main}

Immunotherapy has revolutionized the treatment landscape of many cancers, particularly those with high $\mathrm{TMB}^{1,4-6}$. Somatic mutations can generate antigens (neoantigens) capable of eliciting tumor-specific $\mathrm{T}$ cell responses 7,8 , and it is widely believed that increased TMB renders tumors susceptible to immune attack following ICB treatment. Multiple studies have demonstrated such a correlation and proposed TMB as a predictive biomarker for ICB response ${ }^{9-11}$. In particular, MMRd across all cancers is associated with hypermutation ${ }^{12-16}$ and response to pembrolizumab (anti-PD-1) ${ }^{1}$. However, $50 \%$ of patients with MMRd tumors did not respond and TMB did not stratify responders ${ }^{1}$. This underscores a critical need to understand what factors beyond TMB mediate efficacy. Recent studies have questioned the correlation of TMB with ICB response ${ }^{17}$ and argued that FDA approval of pembrolizumab based on TMB alone may be too broad ${ }^{18}$. Finally, T cell infiltration is a robust predictor of ICB response but is poorly correlated with TMB in pan-cancer analyses ${ }^{19,20}$.

One likely reason for the weak correlation of TMB with ICB response is intratumoral heterogeneity (ITH) of mutations. Neoantigens of low clonality could be deleted with minimal impact on tumor fitness ${ }^{21}$ or fail to elicit productive T cell responses ${ }^{22}$. Indeed, it has been observed in human cancer that ITH is associated with decreased T cell infiltration ${ }^{23,24}$ and poor survival25,26, while clonal neoantigens are predictive of response to $\mathrm{ICB}^{26-28}$. This concept has been exemplified in an experimental setting of UVBinduced hypermutation of melanoma cell lines ${ }^{29}$. It is reasonable to hypothesize that similar mechanisms are operative in MMRd cancers given their constitutive mutational instability. Although previous studies showed that MMRd mutagenesis in vitro renders cell lines immunogenic ${ }^{2,3}$, it is unclear what is the impact of MMR loss in vivo in the presence of immunosurveillance, a process that exquisitely shapes tumor 
immunogenicity $7,30,31$. To address these questions, we developed autochthonous mouse models of sporadic MMRd lung and colon cancer.

Modeling sporadic MMRd in cancer. We adapted the autochthonous mouse model of lung cancer developed in our laboratory ${ }^{32}$ by breeding in a Cas9-expressing allele (R26 LSL-Cas9; Kras ${ }^{\text {LSL-G12D; }}$ Trp53 flox/flox). Intratracheal delivery of lentivirus expressing Cre and an Msh2-targeting sgRNA (sgMsh2) into these animals induced lung adenocarcinomas with efficient MSH2 knockout (Fig. 1a-c, Extended Data Fig. 1a-d). We also adapted an endoscope-guided submucosal injection technique ${ }^{33}$ to deliver lentivirus carrying sgRNAs targeting the colon tumor suppressor, Apc, in tandem with Msh2, Mlh1, Msh3, or Msh6, into the distal colon of mice with constitutive Cas9 expression (Fig. 1b). This efficiently induced focal colon adenomas with MMR gene knockout (Fig. 1d, Extended Data Fig. 1e-i).

To confirm mutation of MMR genes and investigate the degree of TMB, we performed wholeexome sequencing (WES) on micro-dissected tumors at 16-20 weeks post initiation, including 10 sgMsh2and 3 control (sgCtl)-targeted lung tumors, and 5 sgMsh2-, 6 sgMlh1-, 2 sgMsh6-, 6 sgMsh3-, and 5 sgCtltargeted colon tumors. Targeted sequencing of MMR genes in these tumors confirmed extensive frameshifting insertions/deletions (indels). sgMsh2-, sgMlh1-, and sgMsh6-targeted lung and colon tumors showed increased burden of somatic single nucleotide variants (SNVs) and indels, while sgMsh3-targeted colon tumors showed elevated indels only (Fig. 1e-f, Extended Data Fig. 1j). Mutational spectra were Interestingly, one lung tumor had an S415R mutation in the exonuclease domain of DNA polymerase epsilon (Pole) and, consequently, a much higher TMB (Fig. 1e, Extended Data Fig. 1m).

We also performed WES on cell lines derived from sgMsh2-targeted lung tumors. Unexpectedly, these showed a 10-fold greater TMB than sgMsh2-targeted lung tumors (Extended Data Fig. 1j), suggesting either low purity of tumors (unlikely, as all lung tumors were $>78 \%$ pure), mutagenesis in culture, or substantial ITH that was reduced by clonal selection on plastic. Arguing against mutagenesis in vitro, WES of a single cell clone (SSC) revealed more than double the TMB of the parental line, despite a much smaller increase in TMB following 20 passages (Extended Data Fig. 1n). Indicating ITH, sequencing 
Figure 1

a

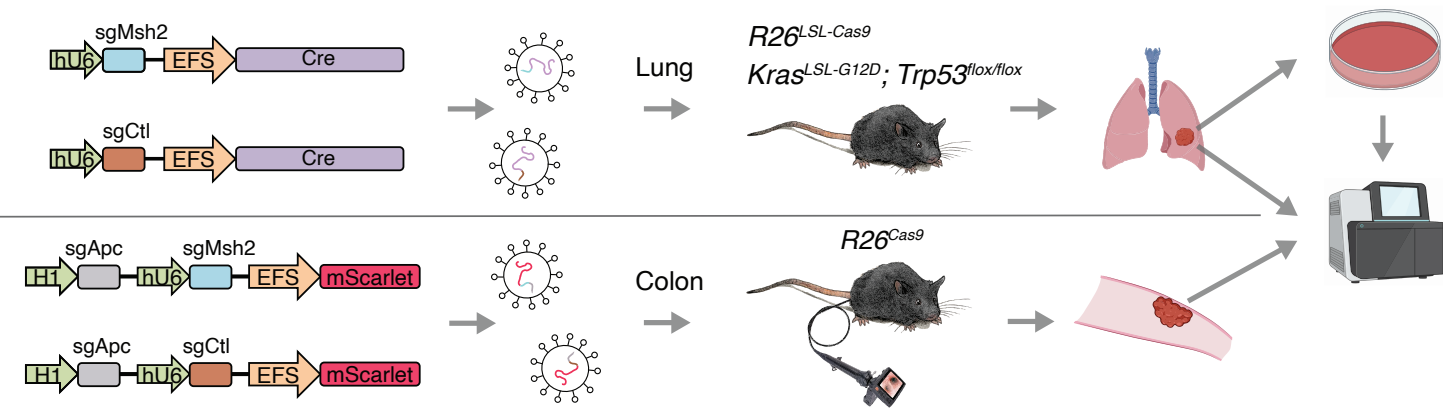

c
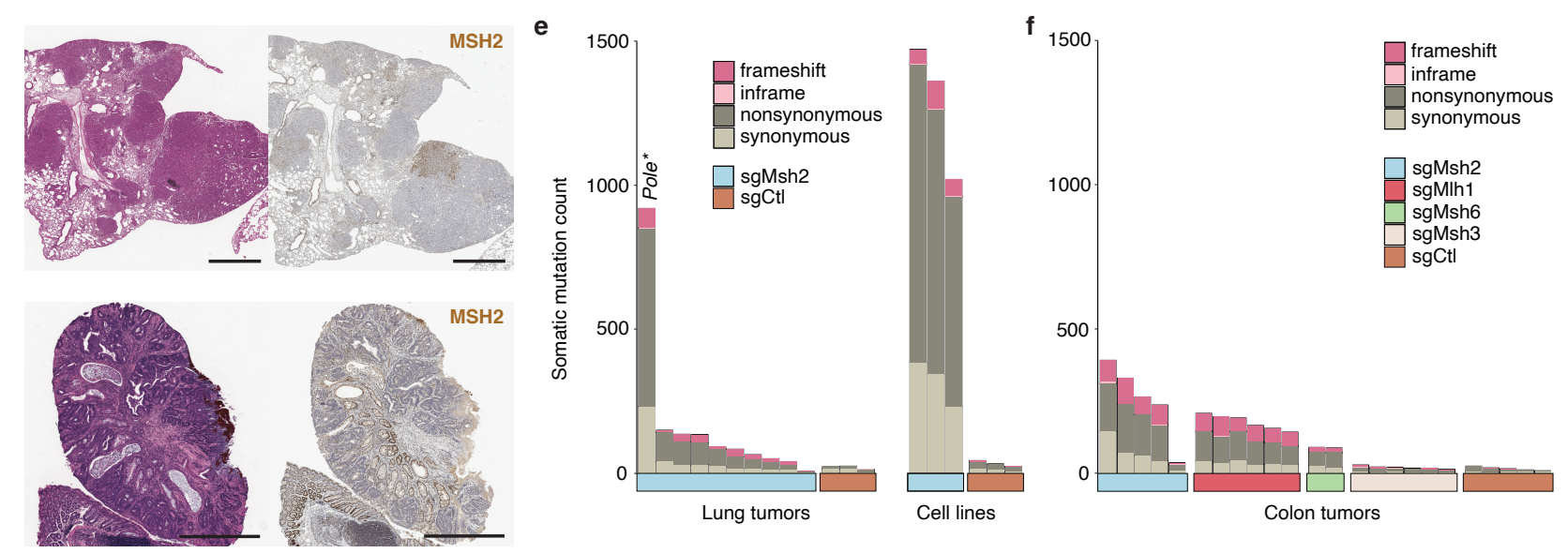

g

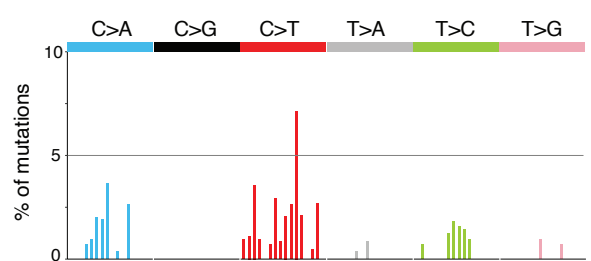

h

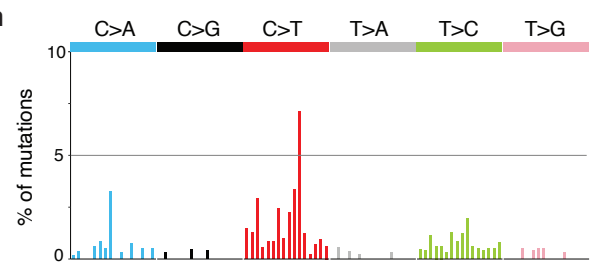

Trinucleotide context and substitution

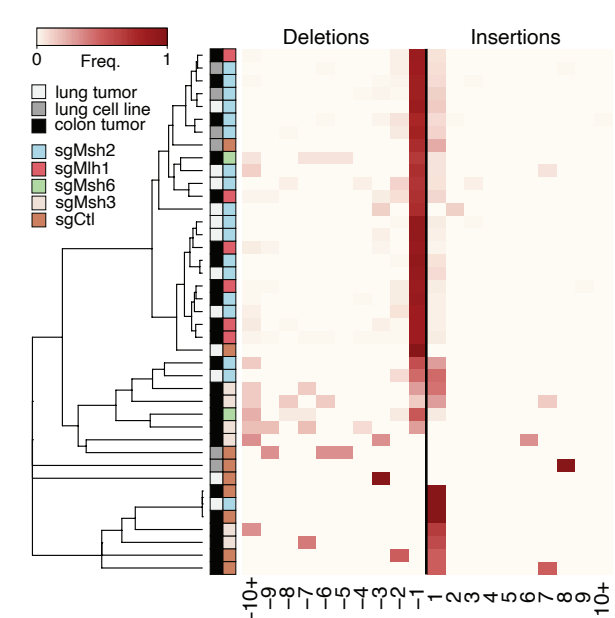

Size

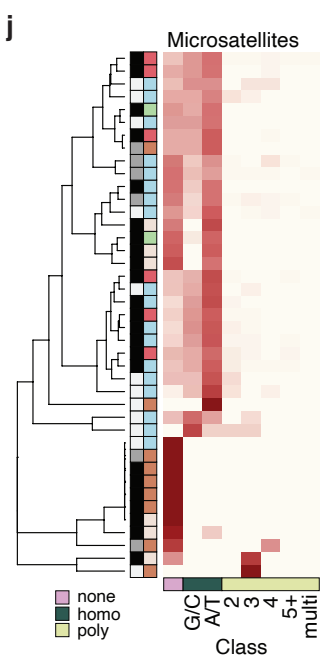


Figure 1 I Development of in vivo CRISPR/Cas9-targeted models of MMRd lung and colon cancer. (a-b)

Schematic of lentiviral constructs and mouse strains used to induce MMRd lung (a) and colon (b) tumors for WES and in vitro analyses. (c-d) H\&E and MSH2 IHC of sgMsh2-targeted lung (c) and colon (d) tumors 16weeks post-initiation, representative of 10 independent animals each. Scale bars $=1 \mathrm{~mm}$. (e-f) Total somatic SNVs (grey shades) and indels (pink shades) identified in the protein coding exome of autochthonous lung tumors and cell lines (e) and autochthonous colon tumors (f). Pole* $=$ Pole S415R mutation. (g-h) Median percentage of the 96 possible SNVs classified by substitution and flanking 5' and 3' bases observed across 9 sgMsh2-targeted lung tumors (excluding one tumor with Pole S415R mutation) (g) and 5 sgMsh2-targeted colon tumors (h). (i-j) Frequency of indels from -10 to 10 nucleotides (i) and the frequency of indels occurring in different DNA microsatellite contexts (j) across all sequenced autochthonous tumors and parental cell lines. In (j), none = no microsatellite, homo $=$ homopolymer runs of four or more $\mathrm{G} / \mathrm{C}$ or $\mathrm{A} / \mathrm{T}$ bases, $2-5+=$ microsatellites with motifs of $2-5+$ bases, and multi $=$ microsatellites with multiple repetitive motifs. 
reads supporting 905 mutations private to the SSC were found in the sequencing data of the parental (but not unrelated control) line, albeit at variant allele frequencies below threshold of mutation calling (Extended Data Fig. 10-p), as previously observed in hypermutated gliomas ${ }^{35}$.

Altogether, these results establish the utility of our models to recapitulate fundamental mutational processes underlying hypermutation in MMRd human cancer. The lower clonal TMB we observed is likely due to neutral evolution in the absence of major selective bottlenecks. Given that ITH is associated with aggressive disease and decreased ICB response in humans ${ }^{25-28}$, these models are uniquely suited to study the role of ITH in immune dysfunction of cancers with high and low prevalence of MMRd alike.

Sporadic MMRd is not immunogenic. Next, we sought to determine the effects of sporadic MMRd on tumorigenesis in our models, including an orthogonal lung model with a Cre-inducible Msh2 knockout allele $^{36}$ (Kras LSL-G12D; Trp53floxfflox; Msh2floxflox_KPM). KPM and MMR proficient control (Kras ${ }^{\text {LSL-G12D; }}$ $\left.\operatorname{Trp53^{flox}/flox}-\mathrm{KP}\right)$ tumors were induced by intratracheal delivery of Cre-expressing adenovirus. MSH2 knockout was slightly more efficient in the KPM (89\%) versus CRISPR/Cas9 (79\%) model (Extended Data Figs. 1a, 2a). Neither model showed a significant difference in overall tumor burden or grade at either time point (Fig. 2a-d, Extended Data Fig. 2b-c). Notably, there was no difference in tumor infiltration by T cells $\left(\mathrm{CD}^{+}\right)$in the sgMsh2-targeted model (Extended Data Fig. 2d-e), or whole lung infiltration by cytotoxic $\left(\mathrm{CD}^{+}\right)$, helper $\left(\mathrm{CD}^{+}\right)$, or regulatory $\left(\mathrm{CD}^{+} / \mathrm{FOXP3}^{+}\right) \mathrm{T}$ cells in the KPM model (Fig. 2e-g), at either time point. Like the lung, tumors in the colon model showed no difference in growth kinetics with Msh2 targeting (Extended Data Fig. 2f).

To determine the sensitivity of these models to immunotherapy, we first performed preclinical trials with ICB (anti-CTLA-4/PD-1) in the KPM model. Additionally, we included treatment with the chemotherapeutic combination of oxaliplatin and low-dose cyclophosphamide (Oxa/Cyc) alone and in combination with ICB (Fig. 2h-i), as Oxa/Cyc has been shown to synergize with ICB37,38. To our surprise, no significant differences were observed between KPM and KP mice across all treatment arms, both in longitudinal change (Fig. 2j) or final tumor burden at necropsy (Fig. 2k). These results were not unique to the lung, as ICB treatment failed to induce any responses in sgMsh2-targeted colon tumors (Fig. 2I-m, 
Figure 2

a

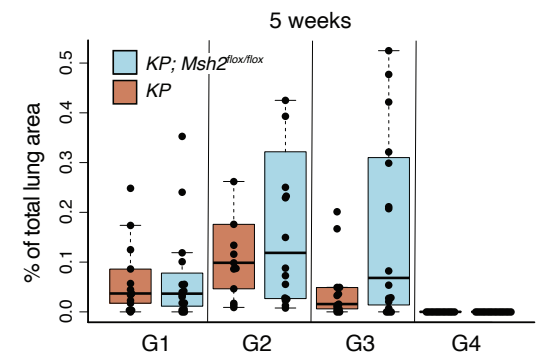

b

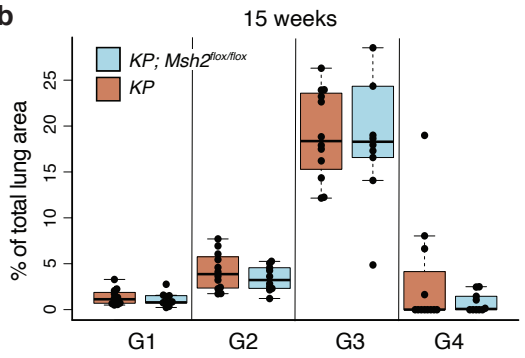

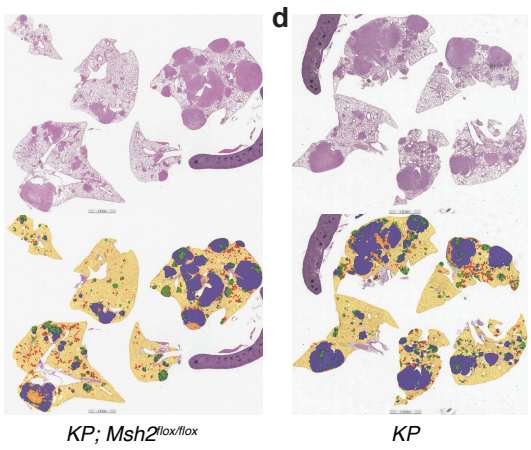

f

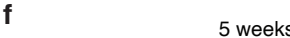

g 15 weeks

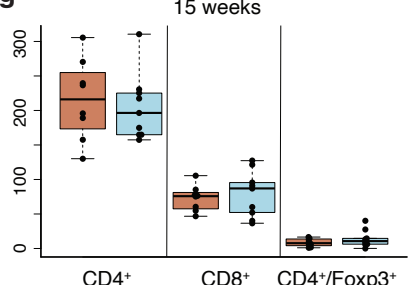

h

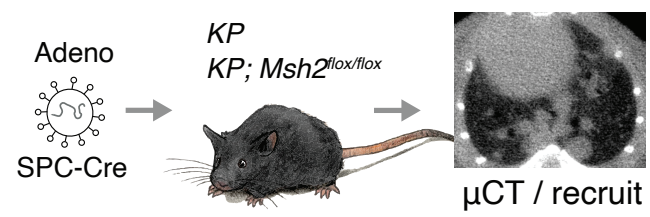

Oxa/Cyc: $x$

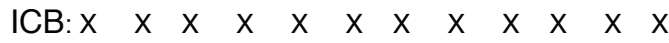

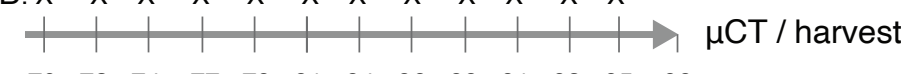

Day: $70 \begin{array}{llllllllllll}72 & 74 & 77 & 79 & 81 & 84 & 86 & 88 & 91 & 93 & 95 & 98\end{array}$

i
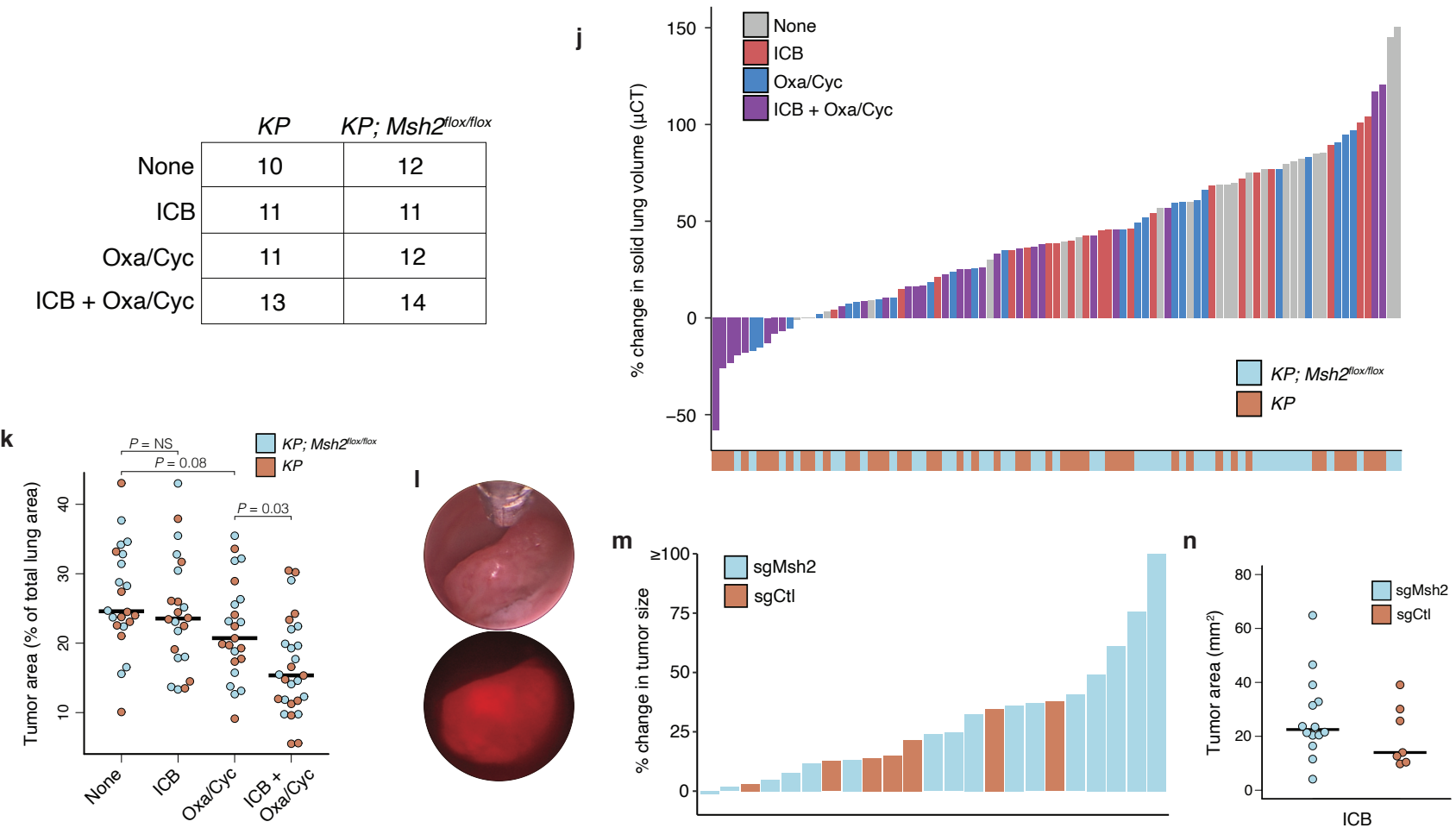
Figure 2 I MMRd models of lung and colon cancer are not immunogenic. (a-b) Percent of total lung area occupied by tumors of grades 1-4 (G1-4) in KP; Msh2floxflox and KP models at 5- (a) and 15-weeks (b) postinitiation with adenovirus expressing Cre driven by the alveolar type II cell-specific surfactant protein C promoter (SPC-Cre), representative of 16 and 15 animals at 5-weeks and 10 and 12 animals at 15-weeks. Normal lung and tumor grades were quantified using an automated convoluted neural network (CNN) developed in collaboration with Aiforia. (c-d) Whole tumor-bearing lung H\&E and CNN annotation masks from $K P ; M s h 2^{f l o x}$ flox $(\mathbf{c})$ and $K P(\mathbf{d})$ models 15-weeks post-initiation, representative of animals in (b). Yellow = normal lung; red = G1; green = G2; blue = G3; orange = G4. (e) IHC staining of lung tumor from 15-week KP; Msh2floxfllox mouse, representative of 10 animals (left). Green $=$ CD4; black $=$ CD8a; red $=$ FOXP3. CD4 ${ }^{+}, \mathrm{CD}^{+}$, and regulatory $\mathrm{T}$ cell annotation masks generated by an automated $\mathrm{CNN}$ developed in collaboration with Aiforia (right). Scale bars $=100 \mu \mathrm{M}$. (f-g) Aiforia CNN quantification of $\mathrm{CD}^{+}, \mathrm{CD}^{+}$, and regulatory (CD4+/FOXP3+) T cell numbers in tumor-bearing lungs of $K P ; M s h 2^{\text {flox/flox }}$ and $K P$ models at 5- (f) and 15-weeks (g) post-initiation, representative of 16 and 15 animals at 5-weeks and 9 and 8 animals at 15-weeks. (h-i) Schematic of preclinical trial design in $K P ; M s h 2^{\text {floxfllox }}$ and $K P$ models (h), and number of animals in treatment arms (i). (j) Change in solid lung volume as measured by $\mu \mathrm{CT}$ pre-treatment (10 weeks) and post-treatment (14 weeks). (k) Lung tumor burden at necropsy (14 weeks) as measured by manual annotation of H\&E-stained whole lung sections. (I) Brightfield and fluorescent colonoscopy images of sgMsh2-targeted colon tumor, representative of 16 animals. Reproducible placement of biopsy forceps in brightfield image allows normalization of relative tumor area. (m) Change in colon tumor size by colonoscopy pre-treatment (20 weeks) and post-treatment (24 weeks). $\mathrm{N}=16$ sgMsh2- and 7 sgCtl-targeted animals. (n) Colon tumor burden at necropsy (24 weeks) as measured by stereomicroscope image. $\mathrm{N}=14$ sgMsh2- and 7 sgCtl-targeted animals. Significance in (k) was assessed by Wilcoxon Rank Sum test. 
Extended Data Fig. 2g-h). Likewise, there was no significant difference in endpoint size of sgCtl- versus sgMsh2-targeted colon tumors following ICB treatment (Fig. 2n). Altogether, these data demonstrate that MMRd is not sufficient to increase sensitivity of tumors to ICB, in stark contrast to previous reports ${ }^{2,3}$.

MMRd drives mutational heterogeneity. To assess the clonal composition of mutations in our models, we estimated cancer cell fractions $(\mathrm{CCF})^{39}$. Most mutations were present in less than a quarter of cells in MMRd tumors from lung and colon (Fig. 3a-b, Extended Data Fig. 3a). To investigate ITH more deeply, we performed WES on 8 SSCs derived from an sgMsh2-targeted lung tumor cell line (09-2). Importantly, prior to subcloning, we restored MMR by re-expressing Msh2 on a bicistronic lentivirus conferring puromycin resistance (Fig. 3c). Of note, these SSCs (M1-8) maintained MSH2 expression and showed stable mutational and clonal architecture after 20 passages in the presence of puromycin (Fig. 3d, Extended Data Fig. 3b-c). Nevertheless, significantly more somatic mutations were called in all SSCs (Fig. 3e), further supporting the notion that ITH is underestimated by bulk sequencing methods ${ }^{35}$. To assess clonal representation of SSCs in the original tumor, we identified unique SSC-defining SNVs and performed ultradeep targeted amplicon sequencing of the parental line (see Methods). This identified three SSCs above background, and only one $>1 \%$ clonal fraction (Fig. 3f). Phylogenetic analysis of SSCs further confirmed a staggering level of ITH (Fig. 3g, Extended Data Fig. 3d).

Given that ITH in our models arose in vivo, we sought to understand the role of immunoediting $7,30,31$ in shaping this process. We performed WES on 10 micro-dissected sgMsh2-targeted lung tumors (week 16-20) from animals continuously depleted of $\mathrm{CD}^{+}$and CD4+ $\mathrm{T}$ cells. Notably, these tumors did not harbor a higher TMB or burden of expressed neoantigens (see Methods) than tumors from immune-competent animals (Fig. 3h-i). However, there was a significant difference in the CCF distribution of neoantigens, with an increased percentage of clonal neoantigens in tumors from T cell-depleted mice (Fig. 3j, Extended Data Fig. 3e). These results argue that neoantigens with high, but not low, clonal fraction are negatively selected by the adaptive immune system. By extension, immunosurveillance in mutationally unstable tumors promotes ITH by increasing the overall fraction of subclonal neoantigens. 


\section{Figure 3}

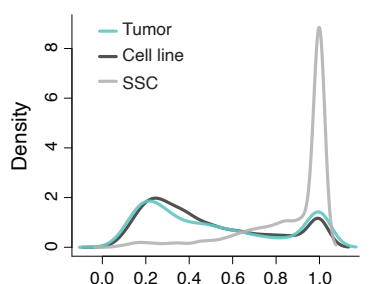

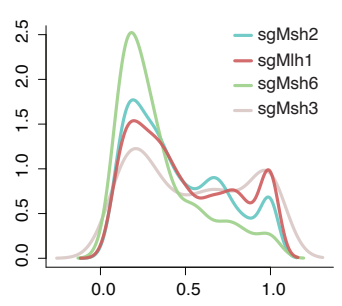

C

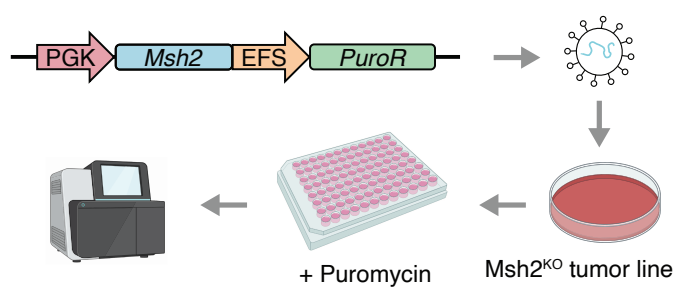

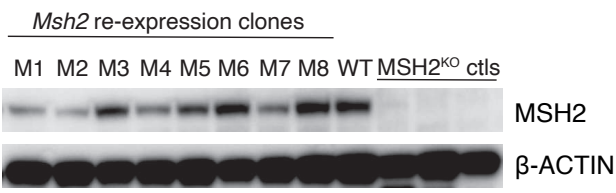

g

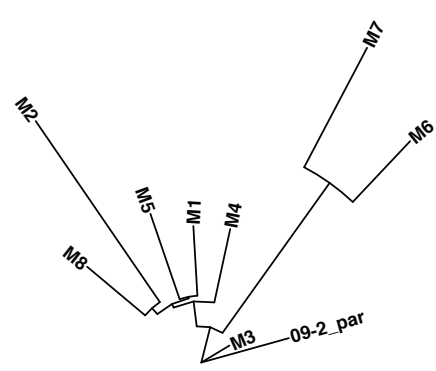

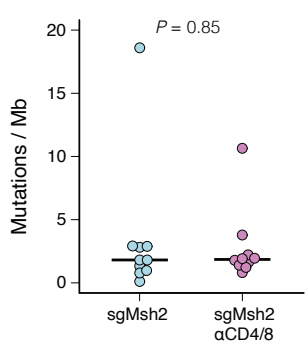

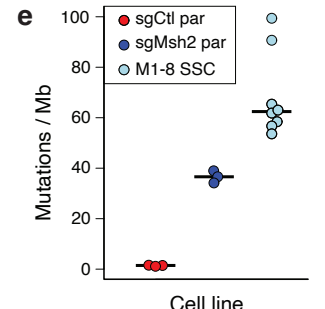

i

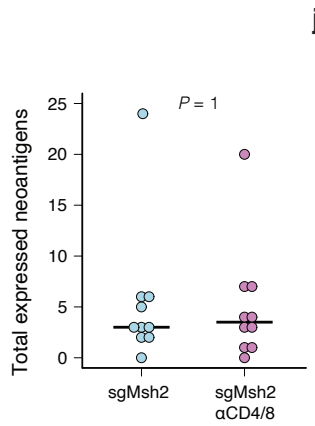

f

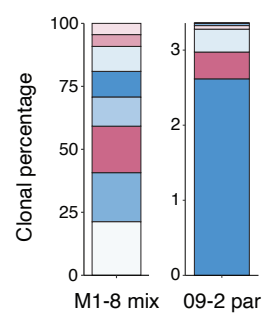

i

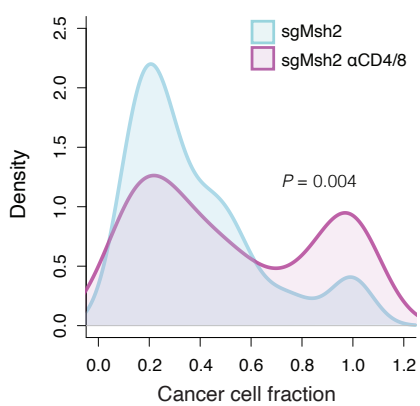


Figure 3 I MMRd models are defined by extensive ITH. (a-b) Distribution of cancer cell fraction estimates of all SNVs in lung tumors, cell lines, and SSCs M1-8 (a) and colon tumors (b). Smoothing was performed by Gaussian kernel density estimation. (c) Schematic of single cell cloning workflow with re-expression of Msh2. (d) Western blot of MSH2 expression in SSCs with Msh2 re-expression. WT = sgCtl-targeted cell line; MSH2 ${ }^{\mathrm{KO}}$ ctls = parental sgMsh2-targeted cell lines. (e) Total mutations identified in ex vivo lung tumor-derived cell lines and SSCs, as mutations / Mb of DNA. Par = parental cell line. $\mathrm{N}=3$ sgCtl, 3 sgMsh2, and 8 M1-8 SSC lines. (f) Estimation of clonal frequencies of M1-8 SSCs in equimolar mixture of SSC DNA (left) and 09-2 parental line (right), as determined by targeted deep amplicon sequencing of 4 private SNVs per SSC. (g) Phylogenetic tree of clonal relationships between M1-8 SSCs, rooted on the parental line 09-2 and constructed using shared mutations with the parsimonious ratchet method. (h-i) Total mutations / $\mathrm{Mb}(\mathbf{h})$ and predicted neoantigens with allele-specific expression > 0 (i) in 16-20-week sgMsh2-targeted autochthonous lung tumors from animals with and without continuous antibody-mediated T cell depletion (aCD4/8). N =10 tumors per group. (j) Distribution of cancer cell fraction estimates of all SNVs in lung tumors from $(\mathrm{h}-\mathrm{i})$. Smoothing was performed by Gaussian kernel density estimation. Significance was assessed by two-sided Kolmogorov-Smirnov test. Significance in (h-i) was assessed by Wilcoxon Rank Sum test. 
ITH masks immunogenicity of subclones. To evaluate the impact of ITH on immunogenicity in our

models, we assessed survival of animals following orthotopic transplantation of lung tumor cell lines and

SSCs described above. Similar to original reports of immunoediting7,30, none of the parental MSH2 knockout lines resulted in accelerated disease with continuous T cell depletion, like the low TMB control line (Fig. 4a). Unlike MMRd in the autochthonous model, however, mice transplanted with the parental line (09-2) showed 22\% durable responses with ICB treatment (Fig. 4b). Transplant of an equal mixture of SSCs M1-8 resulted in modestly accelerated disease with T cell depletion and slightly better response to ICB (Fig. 4c). Transplant of the SSCs individually revealed that three (M3, M7, M8) were strongly immunogenic, resulting in accelerated disease with T cell depletion (Fig. 4d-k). These differences were not due to tumor intrinsic growth rates or defects in antigen presentation, as all SSCs showed similar in vitro growth kinetics and retained interferon gamma (IFNy) sensitivity (Extended Data Fig. 4a-e). Notably, two of the most aggressive and non-immunogenic subclones (M1, M5) were highly responsive to ICB (Fig. 4Im), suggesting that despite minimal baseline immunogenicity, clonal tumors are more sensitive to ICB treatment. Altogether, these results are consistent with decreasing ITH (autochthonous tumors > cell lines > SSC mixture > SSC) correlating with increased immunogenicity and ICB response.

Given that the parental cell line arose in an immune-competent host yet contained highly immunogenic subclones, we reasoned that ITH not only facilitates immune evasion by selective evolution of non-immunogenic subclones but shields immunogenic subclones from deletion. To assess this hypothesis, we collected lung tumors and liver metastases from mice orthotopically transplanted with an equal mixture of SSCs M1-8 and reconstructed their clonal makeup by ultradeep targeted amplicon sequencing (see Methods) (Fig. 4n). Despite the immunogenicity of M3, M7, and M8, these SSCs were detected in five of the seven tumors analyzed from immune-competent animals at clonal fractions $>1 \%$. Two of these animals also developed liver metastases, both of which were clonal outgrowths of M8. In contrast, none of the immune-competent animals transplanted with M3, M7, or M8 alone formed metastases. This pattern was not substantially different than that of transplants in T cell-depleted mice (Fig. 4n). However, ICB treatment resulted in selection against immunogenic subclones, with only one lung tumor harboring an immunogenic subclone $(\mathrm{M} 7)$ at $>1 \%$ fraction, and three liver metastases all 
Figure 4
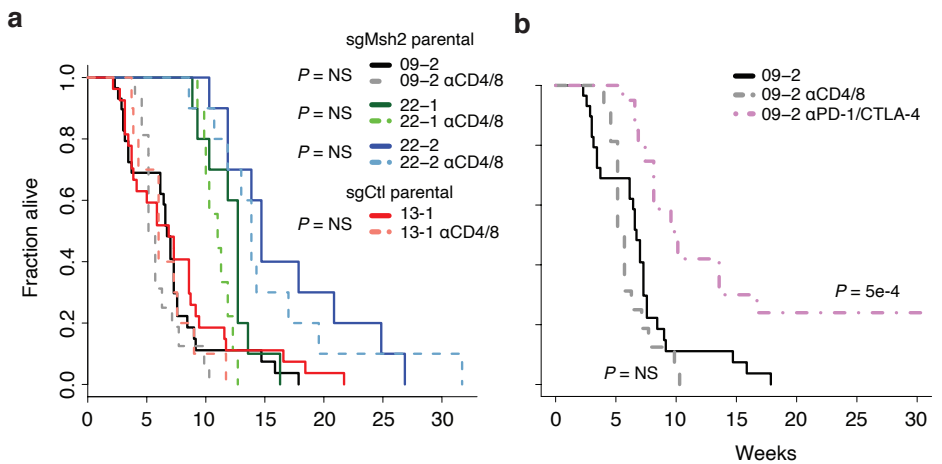

c
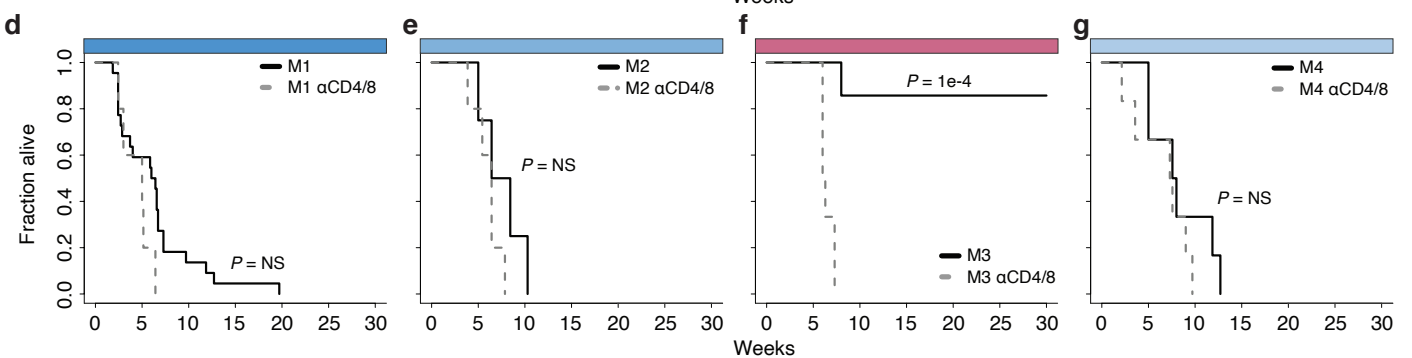

h
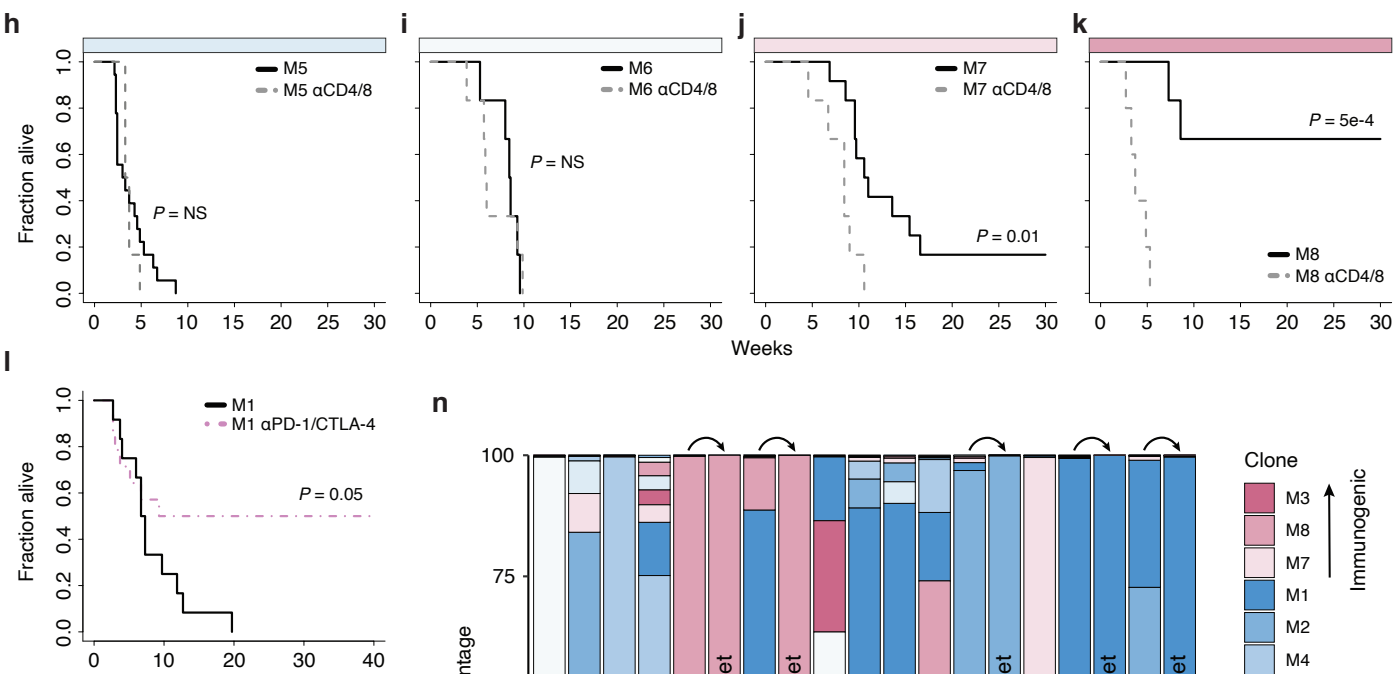

n
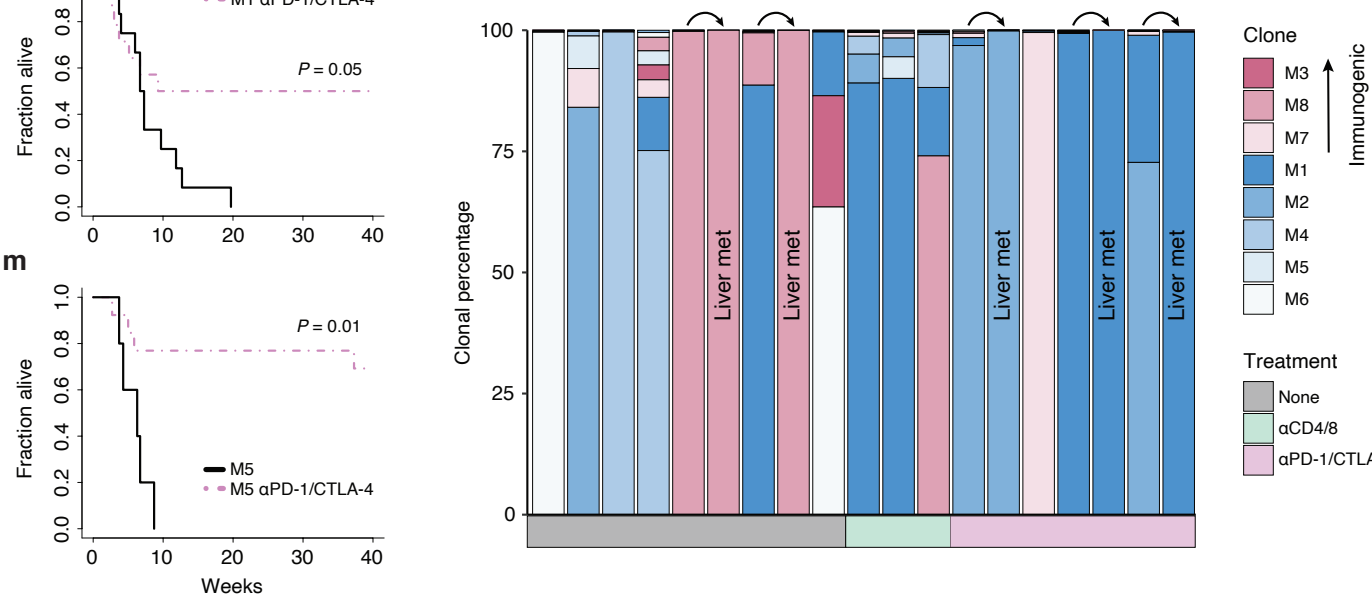

Treatment

$\square$ None

aPD-1/CTLA-4 
Figure 4 I ITH promotes immune evasion of MMRd tumors. (a-n) Survival of syngeneic mice orthotopically transplanted via intratracheal instillation with indicated lung tumor cell lines and SSCs. (a) Transplant with parental sgMsh2- and sgCtl-targeted parental lines, with and without continuous T cell depletion (aCD4/8: lighter shades and dotted lines). (b-c) Transplant with parental 09-2 line (b) and equal mixture of M1-8 SSCs (c) with and without continuous aCD4/8, and ICB treatment. (d-k) Clonal transplants of M1-8 SSCs with and without continuous aCD4/8. (I-m) Transplant of M1 (I) and M5 (m) with and without ICB treatment. Mice that died before initiation of ICB treatment at 2 weeks were excluded from analysis in both arms. (n) Estimation of clonal percentages of M1-8 SSCs in lung tumors and liver metastases from animals transplanted with an equal mixture of all clones and receiving no treatment, continuous aCD4/8, or ICB. Tumors were collected from moribund mice over the course of the survival studies, between 10- and 20-weeks post-transplant for no treatment and ICB arms, and 5- and 7-weeks for aCD4/8 arm. Clonal percentages were determined by targeted deep amplicon sequencing of 4 private SNVs per SSC. Arrows above bars connect matched primary tumors and metastases. ICB treatment in (b-c) and (I-n) was started 2-weeks post-transplant and continued for 4 weeks. Significance in (a-m) was assessed by Cox proportional hazards regression, with Holm's correction for multiple comparisons in (a) and (d-k). 
derived from a single non-immunogenic subclone (Fig. 4n). Altogether, this analysis suggests that not all subclones are intrinsically immune evasive but possess a range of potential immunogenicity that is exposed by ICB treatment. Importantly, sensitivity to ICB appears to be greatest at clonal purity.

\section{Discussion}

Here, we report sporadic MMRd models that recapitulate critical features of human lung and colon cancer, including genetics, histopathology, and in situ initiation in the relevant tissue microenvironment. Unlike previous studies that demonstrated a role for MMRd and TMB in immunotherapy response, in which mutagenesis occurred in vitro, $2,3,29$, our models enable study of mutations continuously acquired in vivo from tumor initiation through advanced disease. Other studies showed immunotherapy efficacy in autochthonous models of spontaneous cancer employing tissue-specific knockout of Msh2 or activation of mutant Pole $\left(\right.$ Pole $\left.{ }^{P 286 R}\right)$ during embryogenesis ${ }^{40,41}$. These models recapitulate familial cancers like Lynch syndrome in the accumulation of mutations in normal parenchyma preceding transformation $(<5 \%$ of CRC), but not sporadic loss of MMR in established tumors $(\sim 10-15 \% \text { of } C R C)^{15,16}$. In contrast, our models did not display increased baseline immunogenicity or response to ICB, likely due to timing of MMR inactivation and resulting patterns of clonal expansion. We induced MMRd concomitantly with tumor initiation, resulting in mutation accumulation during exponential cellular expansion that is reminiscent of so-called 'born to be bad' colon tumors that follow an early explosive growth trajectory ${ }^{42}$. Interestingly, it was recently shown that MMRd occurring de novo in advanced glioma led to extensive ITH and poor ICB response ${ }^{35}$. Tumors in our models developed profound ITH, the full extent of which was not apparent by bulk sequencing, highlighting the importance of standardization of clinical pipelines to estimate TMB. Strategies to robustly assay ITH, such as multi-region or single-cell DNA sequencing, may enhance the predictive utility of TMB.

Similar to another mechanistic study of ITH ${ }^{29}$, we found that experimental reconstitution of ITH potentiated immune evasion. What distinguishes our study is that mutagenesis occurred entirely in vivo. That some of the subclones we isolated were highly immunogenic upon re-transplantation at clonal but not 
subclonal fraction suggests they were protected from deletion by high ITH in the original tumor. This may occur passively due to low cellularity precluding efficient cross priming and driving early $T$ cell dysfunction or ignorance (Westcott, et al, Nature Cancer, 2021, in press) ${ }^{22}$. Alternatively, ITH may generate a multitude of potentially immunogenic neoantigens, but the immune response is directed at a limited subset that is dominant (Burger, et al., Cell, 2021, in press). Paradoxically, we found that immunosurveillance may exacerbate ITH by shaping the clonal architecture of tumors while failing to delete most neoantigens. These results provide nuance to our understanding of immunoediting and highlight the power of our models to capture a hallmark of human cancer that is lacking in carcinogen-induced and geneticallyengineered models alike ${ }^{43}$-the gradual accumulation of mutations over time ${ }^{34}$.

Our results raise important questions related to therapies aimed at deliberately increasing TMB to enhance tumor immunogenicity2,44. These strategies will likely fail to elicit meaningful immune engagement. More concerning, collateral mutagenesis may drive more aggressive cancer, therapy cooperating tumorigenic events will be helpful in determining the impact of clonally selective bottlenecks on immunosurveillance and immunotherapy response. 
218 1. Le, D. T. et al. Mismatch repair deficiency predicts response of solid tumors to PD-1 blockade. Science 357, 409-413 (2017).

2. Germano, G. et al. Inactivation of DNA repair triggers neoantigen generation and impairs tumour growth. Nature 552, 116-120 (2017).

3. Mandal, R. et al. Genetic diversity of tumors with mismatch repair deficiency influences anti-PD-1 immunotherapy response. Science 364, 485-491 (2019).

4. Larkin, J. et al. Five-year survival with combined nivolumab and ipilimumab in advanced melanoma. N. Engl. J. Med. 381, 1535-1546 (2019).

5. Reck, M. et al. Pembrolizumab versus chemotherapy for PD-L1-positive non-small-cell lung cancer. N. Engl. J. Med. 375, 1823-1833 (2016).

6. Fradet, Y. et al. Randomized phase III KEYNOTE-045 trial of pembrolizumab versus paclitaxel, docetaxel, or vinflunine in recurrent advanced urothelial cancer: Results of $>2$ years of follow-up. Ann. Oncol. 30, 970-976 (2019).

7. Matsushita, H. et al. Cancer exome analysis reveals a T-cell-dependent mechanism of cancer immunoediting. Nature 482, 400-4 (2012).

8. Blass, E. \& Ott, P. A. Advances in the development of personalized neoantigen-based therapeutic cancer vaccines. Nature Reviews Clinical Oncology vol. 18 215-229 (2021).

9. Samstein, R. M. et al. Tumor mutational load predicts survival after immunotherapy across multiple cancer types. Nat. Genet. 51, 202-206 (2019).

10. Marabelle, A. et al. Association of tumour mutational burden with outcomes in patients with advanced solid tumours treated with pembrolizumab: prospective biomarker analysis of the multicohort, open-label, phase 2 KEYNOTE-158 study. Lancet Oncol. 21, 1353-1365 (2020). 
244 13. Kim, T.-M., Laird, P. W. \& Park, P. J. The landscape of microsatellite instability in colorectal and endometrial cancer genomes. Cell 155, 858-868 (2013).

14. Chalmers, Z. R. et al. Analysis of 100,000 human cancer genomes reveals the landscape of tumor mutational burden. Genome Med. 9, 1-14 (2017).

15. Cortes-Ciriano, I., Lee, S., Park, W.-Y., Kim, T.-M. \& Park, P. J. A molecular portrait of microsatellite instability across multiple cancers. Nat. Commun. 8, 1-12 (2017).

16. Hause, R. J., Pritchard, C. C., Shendure, J. \& Salipante, S. J. Classification and characterization of microsatellite instability across 18 cancer types. Nat. Med. 22, 1342-1350 (2016).

17. Gurjao, C., Tsukrov, D., Imakaev, M., Luquette, L. J. \& Mirny, L. A. Limited evidence of tumour mutational burden as a biomarker of response to immunotherapy. bioRxiv 2020.09.03.260265 (2020) doi:10.1101/2020.09.03.260265.

18. Rousseau, B. et al. The spectrum of benefit from checkpoint blockade in hypermutated tumors. $N$. Engl. J. Med. 384, 1168-1170 (2021).

19. Spranger, S. et al. Density of immunogenic antigens does not explain the presence or absence of the T-cell-inflamed tumor microenvironment in melanoma. Proc. Natl. Acad. Sci. 113, E7759-E7768 (2016).

20. Cristescu, R. et al. Pan-tumor genomic biomarkers for PD-1 checkpoint blockade-based immunotherapy. Science 362, (2018).

21. McGranahan, N. \& Swanton, C. Clonal heterogeneity and tumor evolution: past, present, and the future. Cell vol. 168 613-628 (2017).

22. Gejman, R. S. et al. Rejection of immunogenic tumor clones is limited by clonal fraction. Elife 7, e41090 (2018).

23. Morris, L. G. T. et al. Pan-cancer analysis of intratumor heterogeneity as a prognostic determinant of survival. Oncotarget 7, 10051-10063 (2016).

24. Lin, Z. et al. Intratumor heterogeneity correlates with reduced immune activity and worse survival in melanoma patients. Front. Oncol. 10, 596493 (2020). 
$27025 . \quad$ Andor, N. et al. Pan-cancer analysis of the extent and consequences of intratumor heterogeneity. Nat. Med. 22, 105-113 (2016).

26. Miao, D. et al. Genomic correlates of response to immune checkpoint blockade in microsatellitestable solid tumors. Nat. Genet. 50, 1271-1281 (2018).

27. McGranahan, N. et al. Clonal neoantigens elicit T cell immunoreactivity and sensitivity to immune checkpoint blockade. Science 351, 1463-1469 (2016).

28. Litchfield, K. et al. Meta-analysis of tumor- and T cell-intrinsic mechanisms of sensitization to checkpoint inhibition. Cell 184, 596-614.e14 (2021).

29. Wolf, Y. et al. UVB-induced tumor heterogeneity diminishes immune response in melanoma. Cell 179, 219-235.e21 (2019).

30. Shankaran, V. et al. IFNgamma and lymphocytes prevent primary tumour development and shape tumour immunogenicity. Nature 410, 1107-11 (2001).

31. DuPage, M., Mazumdar, C., Schmidt, L. M., Cheung, A. F. \& Jacks, T. Expression of tumourspecific antigens underlies cancer immunoediting. Nature 482, 405-9 (2012).

32. DuPage, M., Dooley, A. L. \& Jacks, T. Conditional mouse lung cancer models using adenoviral or lentiviral delivery of Cre recombinase. Nat. Protoc. 4, 1064-1072 (2009).

33. Roper, J. et al. In vivo genome editing and organoid transplantation models of colorectal cancer and metastasis. Nat. Biotechnol. 35, 569-576 (2017).

34. Alexandrov, L. B. et al. Signatures of mutational processes in human cancer. Nature 500, 415-421 (2013).

35. Touat, M. et al. Mechanisms and therapeutic implications of hypermutation in gliomas. Nature 580, $517-523(2020)$

36. Kucherlapati, M. H. et al. An Msh2 conditional knockout mouse for studying intestinal cancer and testing anticancer agents. Gastroenterology 138, 993-1002.e1 (2010).

37. DuPage, M. et al. Endogenous T cell responses to antigens expressed in lung adenocarcinomas delay malignant tumor progression. Cancer Cell 19, 72-85 (2011).

38. Pfirschke, C. et al. Immunogenic chemotherapy sensitizes tumors to checkpoint blockade therapy. 
Immunity 44, 343-354 (2016).

298 39. Zapata, L. et al. Signatures of positive selection reveal a universal role of chromatin modifiers as cancer driver genes. Sci. Rep. 7, 1-15 (2017).

300

40. Gebert, J. et al. Recurrent frameshift neoantigen vaccine elicits protective immunity with reduced tumor burden and improved overall survival in a lynch syndrome mouse model. Gastroenterology 302 (2021) doi:10.1053/J.GASTRO.2021.06.073.

303

41. Li, H.-D. et al. A PoleP286R mouse model of endometrial cancer recapitulates high mutational 304 burden and immunotherapy response. JCI Insight 5, e138829 (2020).

42. Sottoriva, A. et al. A Big Bang model of human colorectal tumor growth. Nat. Genet. 47, 209-216 306 (2015).

43. Westcott, P. M. K. et al. The mutational landscapes of genetic and chemical models of Kras-driven 308 lung cancer. Nature 517, 489-492 (2015).

44. Helleday, T. Making immunotherapy 'cold' tumours 'hot' by chemotherapy-induced mutations-a misconception. Ann. Oncol. 30, 360-361 (2019). 


\section{Extended Data Figure 1}
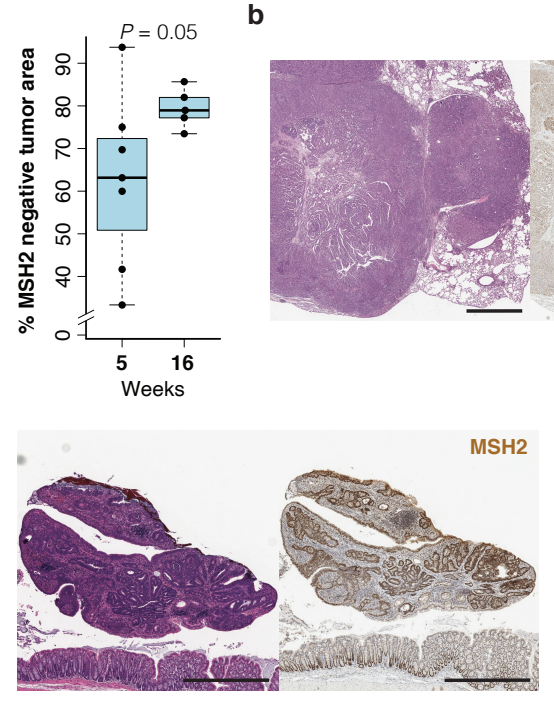

b

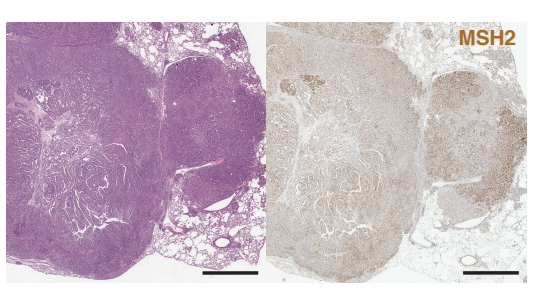

f

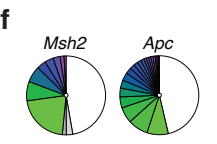

h

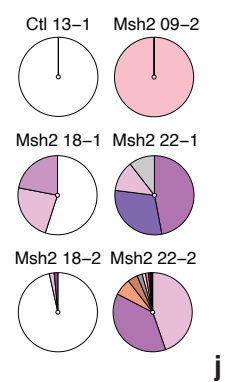

g

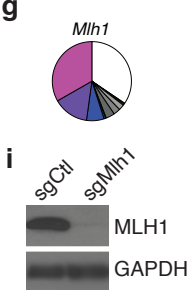

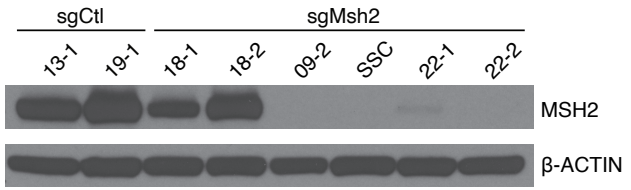

k

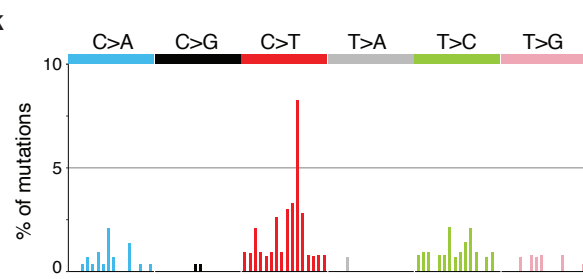

$\begin{array}{lllllll}\text { I } & \mathrm{C}>\mathrm{A} & \mathrm{C}>\mathrm{G} & \mathrm{C}>\mathrm{T} & \mathrm{T}>\mathrm{A} & \mathrm{T}>\mathrm{C} & \mathrm{T}>\mathrm{G}\end{array}$
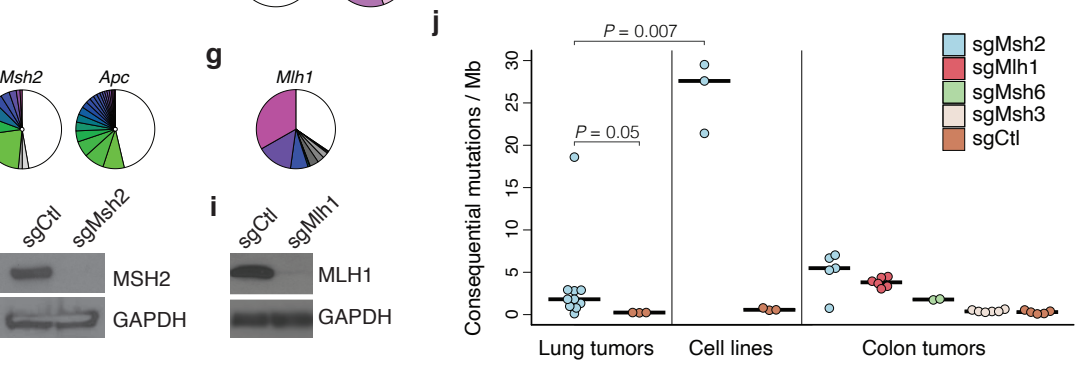

m

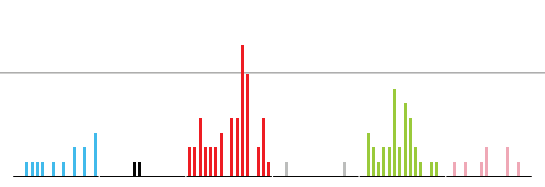

$\mathrm{C}>\mathrm{A} \quad \mathrm{C}>\mathrm{G} \quad \mathrm{C}>\mathrm{T} \quad \mathrm{T}>\mathrm{A} \quad \mathrm{T}>\mathrm{C} \quad \mathrm{T}>\mathrm{G}$

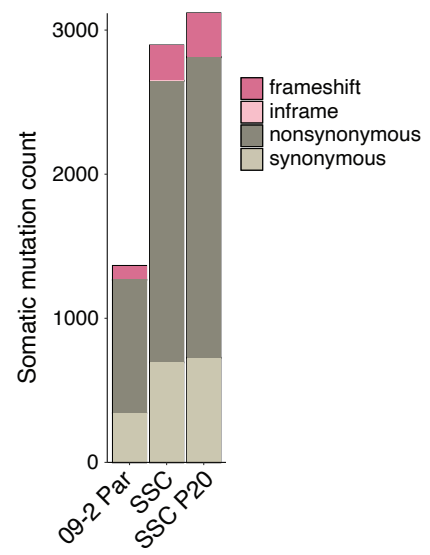

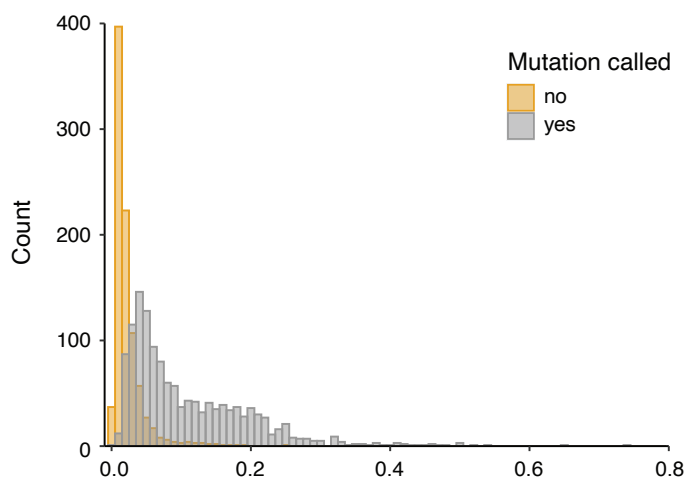

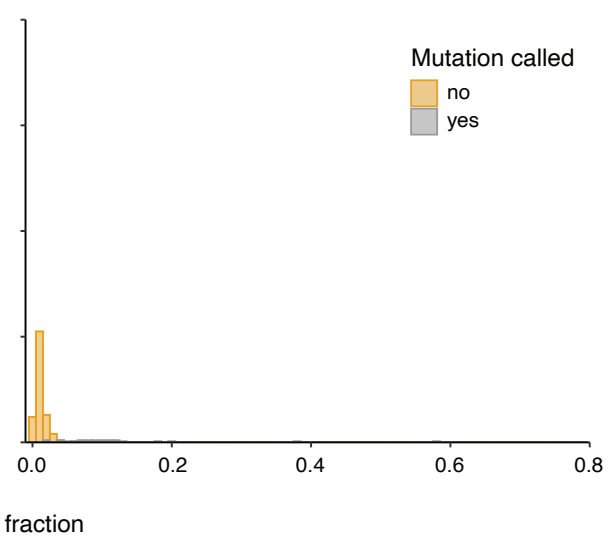


Extended Data Figure 1 I Validation of in vivo CRISPR/Cas9-targeted MMR gene knockout. (a) Percent of overall lung tumor area negative for $\mathrm{MSH} 2$ by $\mathrm{IHC}$ at 5- and 16-weeks post-initiation with sgMsh2 lentivirus. N = 7 animals at 5-weeks and 5 animals at 16-weeks. (b) H\&E and MSH2 IHC of sgCtl (tdTomato)-targeted lung tumors at 16 weeks post-initiation. (c) Amplicon deep sequencing of sgMsh2-targeted locus in cell lines derived from an sgCtl- and five sgMsh2-targeted lung tumors harvested at 16-20 weeks post-initiation. White = unedited (wild-type); Colored = frameshifting mutations; Grey = inframe mutations. (d) Western blot of MSH2 protein expression in sgCtl- and sgMsh2-targeted lung tumor cell lines. SSC = single cell clone derived from 09-2 cell line. (e) H\&E and MSH2 IHC of sgCtl (tdTomato)-targeted colon tumor at 16 weeks post-initiation, representative of 10 animals each. (f-g) Amplicon deep sequencing of sgMsh2/sgApc-targeted (f) and sgMlh1targeted (g) loci in sgMsh2- and sgMlh1-targeted autochthonous colon tumors, representative of 5 sgMsh2and 6 sgMlh1-targeted tumors. Colors same as (c). (h-i) Western blots of MSH2 (h) and MLH1 (i) protein expression in organoids derived from sgCtl-, sgMsh2-, and sgMlh1-targeted colon tumors, representative of one organoid line each. (j) Total consequential mutations (nonsynonymous SNVs and indels) for autochthonous lung tumors and cell lines and autochthonous colon tumors, shown as mutations / Mb of DNA. (k-m) Percentage of the 96 possible SNVs classified by substitution and flanking 5' and 3' base observed in a single sgMsh2-targeted lung tumor with Pole S415R mutation (k) and median percentage across 6 sgMlh1- (I) and 2 sgMsh6-targeted (m) colon tumors. (n) Total somatic SNVs (grey shades) and indels (pink shades) identified in 09-2 lung tumor cell line, early passage SSC derived from 09-2, and the same SSC after 20 passages. (o-p) Total number of mutations identified in early passage SSC that are also supported in sequencing reads of 09-2 parental line (o) and an unrelated MMR proficient control line, 13-1 (p), with variant allele fraction on x-axis. Grey = mutations called by analysis pipeline; gold = mutations not called. Significance in (a) and (j) was assessed by Wilcoxon Rank Sum test. 


\section{Extended Data Figure 2}

a

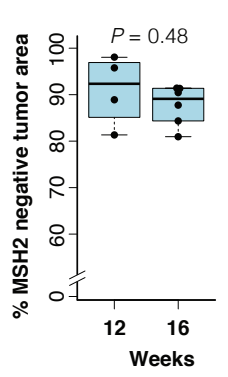

d

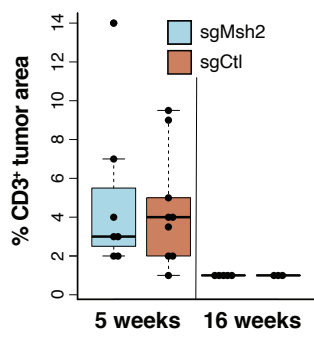

b

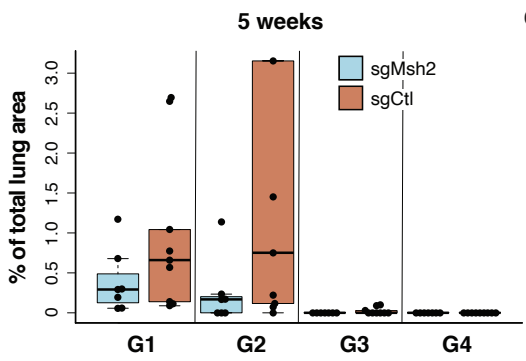

e

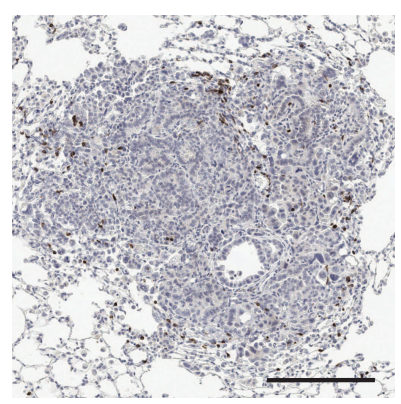

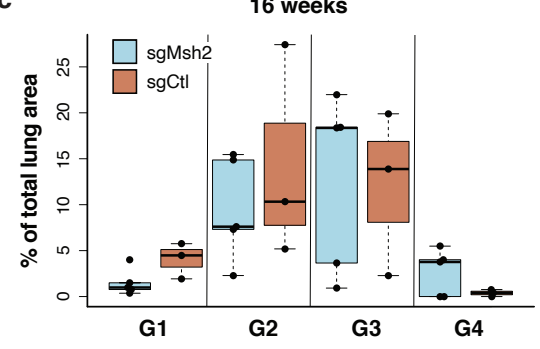

f

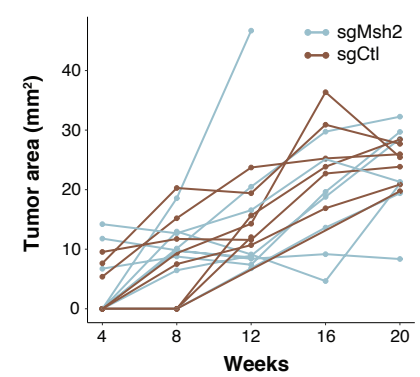

g

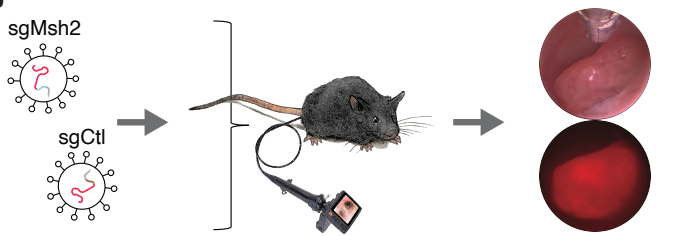

h

\begin{tabular}{|c|c|c|}
\hline & sgCtl & sgMsh2 \\
\hline ICB & 7 & 16 \\
\hline
\end{tabular}

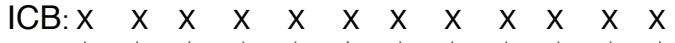

Day: $140142144147149151154156158 \quad 161163165168$ 
Extended Data Figure 2 I Tumor kinetics and immunogenicity are unaffected by MMRd. (a) Percent of overall lung tumor area negative for $\mathrm{MSH} 2$ by $\mathrm{IHC}$ at 12 - and 16-weeks post-initiation of $K P$; Msh2floxflox animals with SPC-Cre adenovirus. $\mathrm{N}=4$ animals at 12-weeks and 6 animals at 16-weeks. (b-c) Percent of total lung area occupied by tumors of grades 1-4 (G1-4) in KP; R26 LSL-Cas9 mice at 5- (b) and 16-weeks (c) post-initiation with sgMsh2- and sgCtl-targeted lentivirus, representative of 7 and 9 animals at 5 -weeks and 5 and 3 animals at 16-weeks. Normal lung and tumors were manually annotated and scored in this cohort. (d) Average lung tumor area positive for CD3, as a marker of all T cells, by IHC in KP; R26 LSL-Cas9 mice at 5- and 16-weeks post-initiation with sgMsh2- and sgCtl-targeted lentivirus. Each point is an independent animal. Number of animals same as in (b-c). (e) IHC staining of CD3 in sgMsh2-targeted lung tumor, representative of animals in (d). Scale bar $=200 \mu \mathrm{M}$. (f) Change in focal colon tumor area over time, as estimated by longitudinal colonoscopy. $\mathrm{N}=8$ sgMsh2- and 8 sgCtl-targeted animals. (g-h) Schematic of colon model preclinical trial design in R26 LSL-Cas9 mice targeted with sgMsh2 and sgCtl (g), and number of animals in each group (h). All animals received ICB treatment. Significance in (a) was assessed by Wilcoxon Rank Sum test. 


\section{Extended Data Figure 3}

a

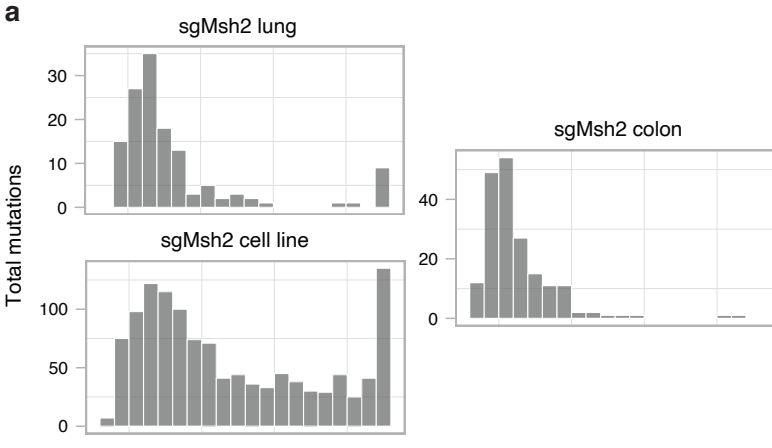

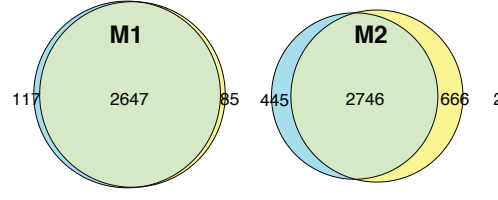

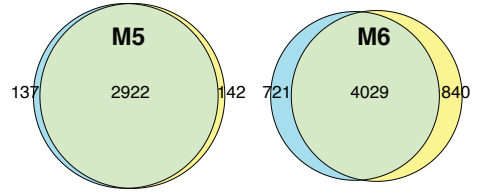

M2
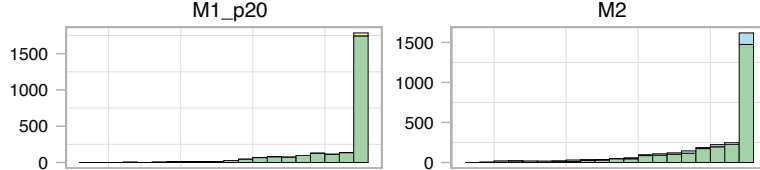

M4
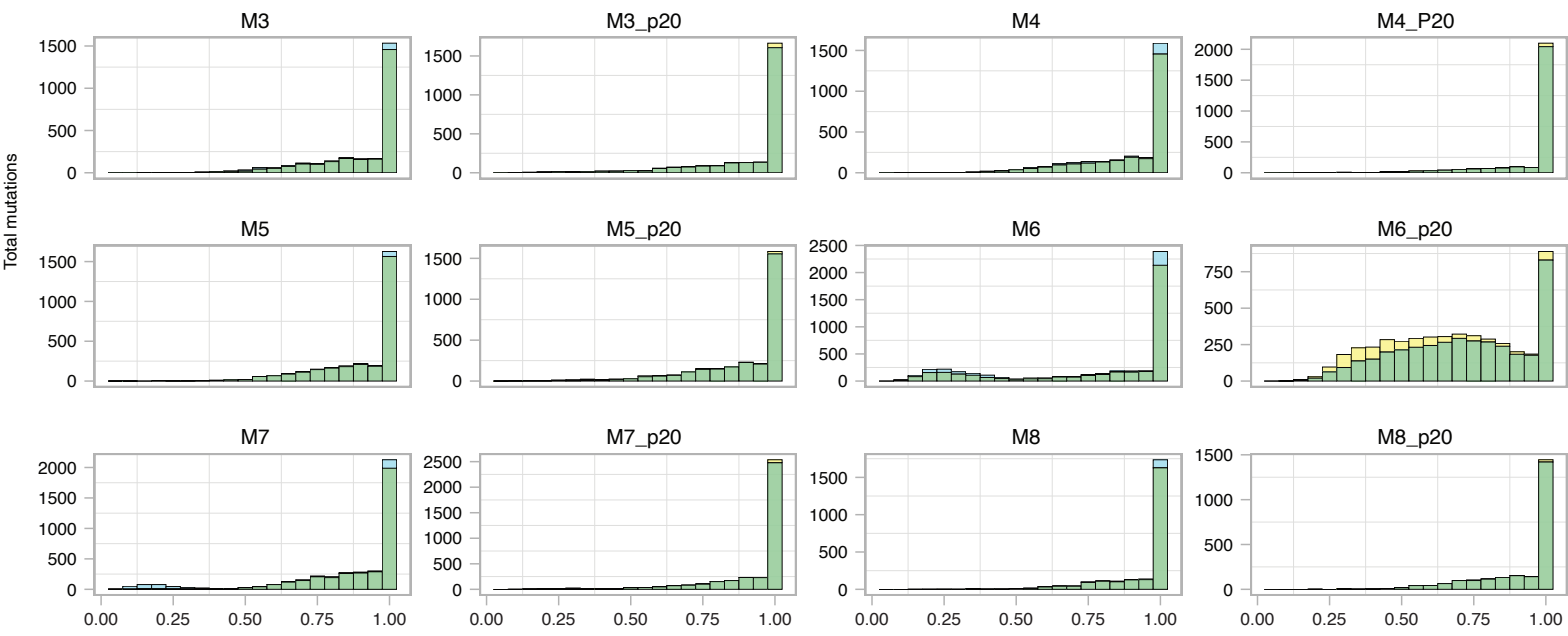

M7 p20
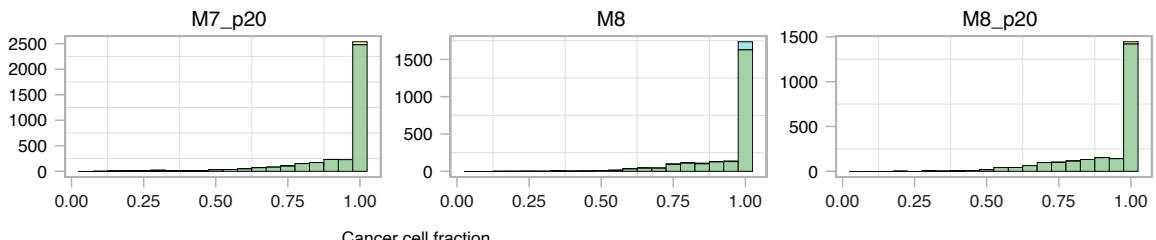

d

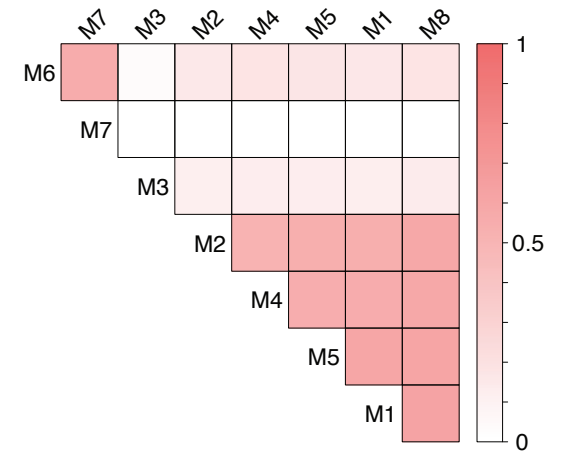

e Passage $0 \square$ Passage $20 \square$ Shared 
Extended Data Figure 3 I SSCs re-expressing Msh2 are mutationally stable. (a) Histograms of total mutations by cancer cell fraction in a representative sgMsh2-targeted lung tumor, cell line, and colon tumor from Fig. 3a-b. (b) Venn diagrams of mutation overlap between M1-8 SSCs sequenced at early passage (called passage 0 for convenience) and 20 passages later. (c) Histograms of total mutations by cancer cell fraction in M1-8 SSCs at passage 0 and 20. (d) Pairwise intersection map of mutations across M1-8 SSCs. Scale represents fraction of total mutations shared between each pair. (e) Distribution of cancer cell fraction estimates of all SNVs in lung tumors from Fig. 3h-j with sgMsh2-targeted Pole S415R mutant lung tumor plotted separately (grey). Smoothing was performed by Gaussian kernel density estimation. P-value represents two-sided Kolmogorov-Smirnov test between sgMsh2 and sgMsh2 aCD4/8 distributions. 


\section{Extended Data Figure 4}

a

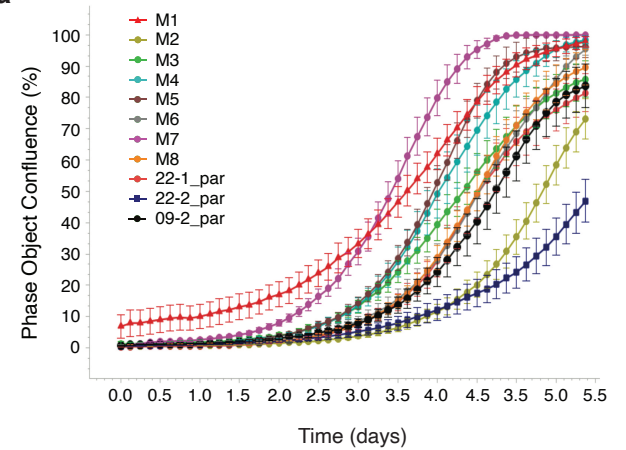

C

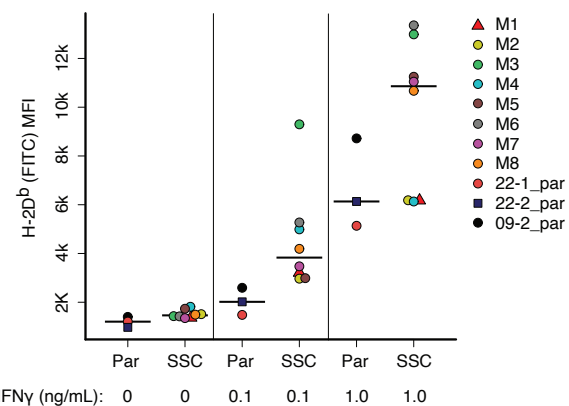

b

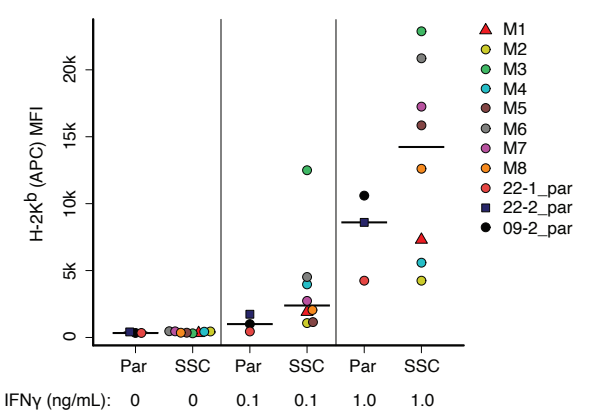

d

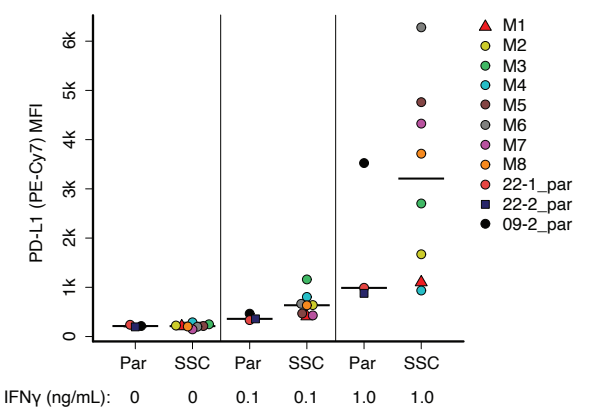

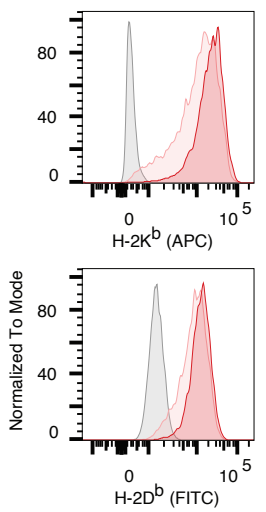

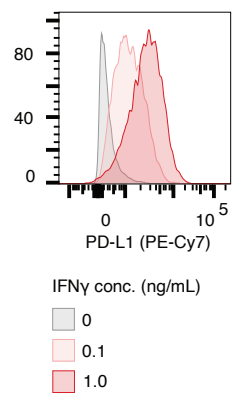


Extended Data Figure 4 I SSCs re-expressing Msh2 grow similarly in vitro and are IFNy responsive. (a)

In vitro growth kinetics of parental MSH2 knockout cell lines (Par; 09-2, 22-1, 22-2) and Msh2 re-expressing SSCs generated from 09-2 (M1-8), measured by live cell imaging with an IncuCyte S3. Bars represent standard deviation of eight independent replicates (wells). (b-e) Flow cytometric analysis of surface expression of $\mathrm{MHC}-\mathrm{I}$ alleles $\mathrm{H}-2 \mathrm{~K}^{\mathrm{b}}$ (b) and $\mathrm{H}-2 \mathrm{D}^{\mathrm{b}}$ (c) and IFNY-response gene PD-L1 (d) in cell lines from (a) following overnight stimulation with $0,0.1$, and $1.0 \mathrm{ng} / \mathrm{mL} \mathrm{IFN \gamma}$. MFI = mean fluorescence intensity. (e) Representative histograms of $\mathrm{H}-2 \mathrm{~K}^{\mathrm{b}}, \mathrm{H}-2 \mathrm{D}^{\mathrm{b}}$ and PD-L1 expression in an SSC (M3) from the experiment in (b-d). 


\section{Methods}

\section{Mice}

Mice were housed in the animal facility at the Koch Institute for Integrative Cancer Research at MIT with a 12hour light/12-hour dark cycle with temperatures within $68-72^{\circ} \mathrm{F}$ and $30-70 \%$ humidity. All animal use was approved by the Department of Comparative Medicine (DCM) at MIT and the Institutional Animal Care and Use

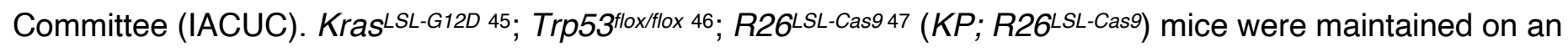
F1 (C57BL/6 x 129/SvJ) background. Kras LSL-G12D; Trp53flox/flox; Msh2floxflox 36 (JAX stock \#016231) and R26 Cas9 48 (JAX stock \# 028555) mice were maintained on a pure C57BL/6 background. Lung cell lines were isolated from tumors induced in albino C57BL/6 hosts chimeric for tissue derived from blastocyst injection of a $K P$; R26LSL-Cas9 embryonic stem (ES) cell line (12A2) of mixed C57BL/6 and 129/SvJ background and male sex, as previously described ${ }^{47}$. These mice were used in early experiments prior to establishment of a germline $K P$; R26 $6^{\text {LSL-Cas9 }}$ colony. In orthotopic lung studies, cell lines were transplanted into male chimeras generated from the same 12A2 ES cell line at 10-16 weeks of age. These chimeras are tolerized to C57BL/6 and129/SvJ tissues, potential antigens in the R26 LSL-Cas9 allele, and PuroR introduced into cell lines with Msh2 reexpression (Kras ${ }^{L S L-G 12 D}$ tissue in the unrecombined state contains PuroR in the lox-STOP-lox cassette). Autochthonous tumors in lung and colon were induced in approximately equal numbers of male and female mice at 8-16 weeks of age.

\section{Tumor models}

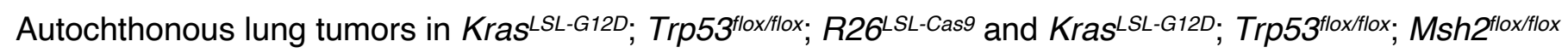
mice were induced by intratracheal instillation of $2 \mathrm{e} 4$ transduction units (TUs) of lentivirus and $2 \mathrm{e} 8$ plaque forming units of adenovirus, respectively, as previously described ${ }^{32}$. Autochthonous colon tumors in $R 26^{\text {Cas } 9}$ mice were induced by endoscope-guided submucosal injection in the distal colon, as previously described ${ }^{33,49}$. Two injections at $1.5 \mathrm{e} 6$ TUs of lentivirus in $50 \mu \mathrm{L}$ OPTI-MEM were delivered per mouse. Lentivirus was 
produced in HEK-293 cells (ATCC) and concentrated as previously described ${ }^{32}$, and functional titers (Cre activity, mScarlet fluorescence) measured as previously described ${ }^{50}$. Cell lines were orthotopically transplanted by intratracheal instillation of 1 e 5 cells in $50 \mu \mathrm{L}$ SMEM with $5 \mathrm{mM}$ EDTA, followed by a rinse of $30 \mu \mathrm{L}$ with the same media. Cell lines were established from autochthonous lung tumors by microdissection and mechanical mincing in digestion buffer (HBSS with 1 M HEPES, 125 Units/mL Collagenase Type IV (Worthington) and 20 $\mu \mathrm{g} / \mathrm{mL}$ DNAse (Sigma-Aldrich) followed by incubation at $37^{\circ} \mathrm{C}$ with gentle agitation for $30 \mathrm{~min}$, and plating in RPMI 10\% FBS. Lines were plated into 50:50 RPMI/DMEM 10\% FBS at first passage, and DMEM 10\% FBS at second passage and thereafter. Cells were taken for WES at third passage. Msh2 expressing lentivirus was produced as above but without concentration. Cells were incubated with lentiviral supernatant and 3 days later selection begun with $6 \mu \mathrm{g} / \mathrm{mL}$ puromycin (Thermo Fisher), which was maintained during all subsequent culturing.

\section{Whole-exome sequencing analysis}

Whole-exome libraries for all samples were generated using the SureSelect XT Mouse All Exon (Agilent) target enrichment kit with 100 bp paired-end sequencing on the Illumina HiSeq 4000 platform, except for the M1-8 passage 20 SSCs, which were 150 bp paired-end sequencing on the Illumina NovaSeq 6000 S4 platform. Library preparation and sequencing to 100X on-target coverage was performed by Psomagen. Raw sequencing reads were mapped to the GRCm38 build of the mouse reference genome using BWA-MEM v0.7.17-r118851. Aligned reads in BAM format were processed following the Genome Analysis Toolkit (GATK) v4.1.8.052 Best Practices workflow to remove duplicates and recalibrate base quality scores.

SNVs and indels were detected using Mutect2, MuSE v1.0rc ${ }^{53}$, VarDict v1.8.2 ${ }^{54}$, and Strelka2 v2.9.2 ${ }^{55}$ using matched normal tails as controls. Mutect2 was run using a panel of normals generated using the sequencing data for the 11 tails analysed in this study. Each caller was run independently on each tumor-normal pair, and the calls were integrated using SomaticCombiner ${ }^{6}$. For the colon tumors, a panel of four normal tails was used to generate the normal control for all samples, as these mice were of a pure background. We only considered those SNVs detected by Mutect2 and supported by at least one of the other callers. To increase the 
accuracy of indel detection, only indels detected by at least 2 algorithms were considered for further analysis. Variants mapping to DbSNP (build id 150) positions were discarded. Microsatellite contexts of mutations were annotated using SciRoKo v3.457, with minimum score $=8$, seed length $=8$, repeats $=2$, and mismatch penalty $=1$. Only mutations within exons were considered in all analyses.

Somatic copy number aberrations were detected by integrating the output of GATK and FreeBayes v1.358 using PureCN v1.16.059. Briefly, the GATK4 Somatic CNV workflow was utilized for the normalization of read counts and genome segmentation using the tails of all samples to build the panel of normals. FreeBayes was used to obtain B-allele frequency values for dbSNP (build id 150) variant sites of the mouse genome. Finally, PureCN was used to integrate the output of GATK and FreeBayes to estimate the allele-specific consensus copy-number profile, purity, and ploidy of each sample. Ploidy values of cell lines were determined experimentally by metaphase spreads and input into PureCN. Finally, the cancer cell fraction (CCF) value for each SNV was computed using the R package cDriver ${ }^{39}$.

\section{Clonal deconvolution by targeted amplicon sequencing}

To identify private somatic SNVs capable of distinguishing individual SSCs in the parental cell line (09-2_par) and M1-8 SSC mixed tumors (Fig. 3f, 4n), we first selected all clonal SNVs in copy neutral regions of the genome (4 copies, as all lines were tetraploid by metaphase spreads). We then checked the BAM files across all other samples for the presence of reads supporting the alternative allele (base quality $>20$ and mapping quality $>30$ ) using an in-house python script relying on the Pysam library. Mutations with support for the alternate allele in at least one sample were discarded. Four private SNVs for each SSC and four common SNVs were chosen for targeted amplicon sequencing, all of which were validated by PCR amplification and Sanger sequencing. 200250 bp regions spanning these SNVs were individually PCR amplified from samples, gel purified, and submitted for 150 bp paired-end sequencing on the Illumina NovaSeq 6000 S4 platform.

Reads were aligned to a reference fasta file of all targets (+/- $250 \mathrm{nt}$ upstream/downstream of SNV in mouse genome, build GRCm38) using BWA-MEM, with removal of duplicates and recalibration of base quality score following GATK Best Practices. A pileup was then generated using the mpileup function of bcftools 
v1.10.2 $2^{60}$ with --min-BQ 30 and a bed file of all SNV coordinates. Using a custom R script, total and SNV-specific depths at all locations were extracted. All SNVs showed more reads supporting the expected alternate allele than other alleles in the M1-8 SSC equal mixture control, except for M6_2, which was excluded from subsequent analysis. Background PCR/sequencing error for each SNV was estimated using the median observed frequencies of SNVs in all metastases (excluding those of the single represented clone in each metastasis), which represented truly clonal controls. SNV frequencies were adjusted by subtracting background values. Clonal percentages in ex vivo tumors were estimated by taking the median of private SNV frequencies, multiplying by 4 (SNVs are present on 1 of 4 alleles), and dividing by tumor purity. Tumor purity was estimated by taking the median observed to expected ratio of frequencies of the four common SNVs (present in all SSCs).

\section{Neoantigen prediction and expression}

Variant consequence was annotated using Ensembl Variant Effect Predictor (VEP) v9961 with Wildtype and Downstream plugins, the VEP cache and reference genome for GRCm38, and the following parameters: -symbol, --terms=SO, --cache, --offline, --transcript_version, --pick. The --pick parameter was reordered from default to report the transcript with most extreme consequence for each variant: rank, canonical, appris, tsl, biotype, ccds, length, mane. Neoepitopes were predicted with C57BL/6 mouse MHC-I alleles, $H 2-K_{1}\left(\mathrm{H}-2 \mathrm{~K}^{\mathrm{b}}\right)$ and $H 2-D 1\left(H-2 D^{b}\right)$, and variant effect predictions using pVACtools v1.5.762. Mutant peptides were generated for lengths 8- through 11- amino acids, and MHC:peptide binding affinity was predicted for all peptide:MHC allele pairs with NetMHC-4.0, NetMHCpan-4.0, SMM v1.0, and SMMPMBEC v1.063-66. The median value across all affinity predictions was taken as the final measure of binding affinity.

To assess allele-specific expression of neoantigens, RNA-seq was performed on autochthonous lung tumors (10 sgMsh2 and 10 sgMsh2 with aCD4/8 treatment) and M1-8 SSCs. cDNA libraries were prepared using Kapa mRNA Hyperprep, and 150 bp paired-end sequencing was performed on the Illumina NextSeq platform. Sequencing reads were aligned to the reference genome (GRCm38) using STAR v2.7.12 ${ }^{67}$ and PCR duplicates were removed using Picard v2.23.468. STAR parameters used: outFilterMultimapNmax $=20$, alignSJoverhangMin $=8$, alignSJDBoverhangMin $=1$, outFilterMismatchNmax = 999, 
outFilterMismatchNoverLmax $=0.1$, alignIntronMin $=20$, alignIntronMax $=1000000$, alignMatesGapMax $=$ 1000000, outFilterScoreMinOverLread $=0.33$, outFilterMatchNminOverLread $=0.33$, and limitSjdblnsertNsj $=$ 1200000. Considering the somatic variants found in each sample by WES, we used a custom python script to interrogate the presence of these variants in the RNA-seq BAM files. Only non-duplicate reads with mapping quality $>=255$ and bases with base-quality $>=20$ were considered to compute the variant allele frequencies in RNA.

\section{Immunohistochemistry}

Tissues were fixed in zinc formalin, washed in $70 \%$ ethanol and paraffin embedded. Antigen retrieval was performed in citrate buffer $\mathrm{pH} 6$ in a pressure cooker at $125^{\circ} \mathrm{C}$ for five minutes. Blocking was performed with BLOXALL Endogenous Peroxidase and Alkaline Phosphatase Blocking Solution (Vector) followed by Normal Horse Serum (2.5\%) (Vector). For MSH2 (ab70270, Abcam) and CD3 (ab5690, Abcam) stains, slides were stained overnight at 1:1000, then incubated with HRP anti-Rabbit IgG (Vector) and developed with DAB (Vector). For triple staining (CD8a, CD4, FOXP3), slides were first stained with CD8a (ab217344, Abcam) 1:1000 overnight, incubated with Alkaline Phosphatase (AP) anti-Rabbit IgG (Vector) and developed with Vector Black substrate (Vector). Sections then underwent a second round of antigen retrieval in a pressure cooker at $110^{\circ} \mathrm{C}$ for two minutes, followed by co-incubation with FOXP3 (FJK-16s, eBioscience) 1:125 and CD4 (ab183685, Abcam) 1:400 overnight. Sections were then sequentially incubated with AP anti-Rat lgG (Vector) and HRP anti-Rabbit IgG and developed sequentially with Vector Red (Vector) and Vina Green (Biocare Medical). Slides were counterstained with Harris Acidified Hematoxylin and dehydrated. Aqueous wash steps following counterstain were shortened from 1 minute to 30 seconds to minimize loss of Vina Green stain.

\section{Quantification of tumor burden and immunohistochemistry staining}


Quantification of lung tumor burden by grade was performed on scans of hematoxylin and eosin (H\&E)-stained sections by an automated deep neural network developed in collaboration with Aiforia Technologies Oy, and in consultation with veterinarian pathologist Dr. Roderick Bronson. A convolutional neural network (CNN) for semantic multi-class segmentation was trained to classify and detect lung parenchyma and non-small cell lung cancer (NSCLC) grades 1-4. For supervised training, selected areas from 93 slides were chosen. The algorithm performed consistently and with high correlation with human graders across multiple validation datasets independent of the training dataset. Version NSCLC_v25 of the algorithm was used.

Immune infiltration in triple stained slides was calculated by a CNN trained to identify the three cell types stained $($ black $=$ CD8, green $=$ CD4, green/red $=$ Treg), in collaboration with Aiforia Technologies Oy . Whole slides were scanned with a Leica AT2 (Aperio) using the Rainbow color profile. First, the CNN was trained to identify a tissue layer. Within that layer, the CNN was trained to identify black, green, and green/red staining. Within each of these layers, an object counter was trained to quantify the number of cells with the stain. Training was performed by manual annotation of each layer and counting of objects within training regions across 20 separate slides, with roughly five training regions per layer per slide. Performance was validated against human counting and found to be highly accurate and consistent. CD3 infiltration in single stain slide scans was measured as the percentage of pixels positive for stain (DAB) in Aperio ImageScope. Area of positive and negative MSH2 staining was quantified by manual annotation in QuPath v0.1.269.

\section{Western blot}

Cells were lysed in RIPA buffer (Thermo Fisher) supplemented with Halt protease inhibitors (Thermo Fisher) and incubated for $30 \mathrm{~min}$ at $4{ }^{\circ} \mathrm{C}$ rotating. Protein concentration was determined using Pierce BCA Protein Assay (Thermo Fisher), and equal amounts of protein $(20-40 \mu \mathrm{g})$ were run on NuPage 4-12\% Bis-Tris gradient gels (Thermo Fisher) by SDS-PAGE and transferred to PVDF membranes. Immunoblotting was performed against MSH2 (D24B5, Cell Signaling Technology) at 1:1000, MLH1 (ab92312, Abcam) at 1:1000, GAPDH (6C5, Santa Cruz) at 1:5000, and $\beta-A C T I N ~(13 E 5$, Cell Signaling Technology) at 1:5000. Blots were stained with HRP anti-Rabbit IgG and developed with Western Lightning Plus-ECL (Perkin Elmer) on X-ray film. 


\section{In vivo antibody and chemotherapy dosing}

All antibody dosing was performed via intraperitoneal injection in $100 \mu \mathrm{PBS}$. aCD4 (GK1.5, BioXCell) and aCD8 (2.43, BioXCell) depleting antibodies were administered at 200 ug every 4 days. aPD-1 (29F.1A12, BioXCell) was administered at $200 \mu \mathrm{g}$ three times a week. aCTLA (9H10, BioXCell) was administered at an initial dose of $200 \mu \mathrm{g}$, with all subsequent doses at $100 \mu \mathrm{g}$, three times a week. Oxaliplatin (Sigma) and cyclophosphamide (Sigma) (Oxa/Cyc) were co-delivered via intraperitoneal injection in $100 \mu$ PBS at 2.5 $\mathrm{mg} / \mathrm{kg}$ and $50 \mathrm{mg} / \mathrm{kg}$ body weight, respectively, once a week for three weeks, as previously described ${ }^{38}$.

\section{In vivo tumor imaging and quantification}

Lung tumor progression was monitored longitudinally by X-ray microcomputed tomography $(\mu C T)$ using a GE eXplore CT 120 system, as previously described ${ }^{70}$. Solid lung volume corresponding to tumor burden was quantified using a custom MATLAB (MathWorks) script, as previously described ${ }^{70}$. Colon tumor progression was monitored longitudinally using a Karl Storz colonoscopy system with white light and RFP fluorescence. This consists of Image 1 H3-Z Spies HD Camera System (part TH100), Image 1 HUB CCU (parts TC200, TC300), 175-Watt D-Light Cold Light Source (part 20133701-1), AIDA HD capture system, and fluorescent filters in the RFP and GFP channels (Karl Storz). The endoscope used for imaging was the Hopkins Telescope (Karl Storz, part 64301AA) with operating sheath (Karl Storz, part 64301AA). To consistently measure tumor area, biopsy forceps (Richard Wolf) were fed through the operating sheath and positioned consistently given two landmarks: widthwise grooves that appear as concentric semi-circles in the field of view, and a lengthwise groove at the forceps tip. Images were captured upon gentle contact of forceps with tumor. Tumor area in the field of view and length of the lengthwise forceps groove were calculated using ImageJ v2.1.0/1.53c. Tumor area was normalized to groove length.

\section{Generation of lentiviral constructs}


The previously published U6::sgRNA-EFS::Cre (pUSEC) lentiviral construct ${ }^{70}$ was digested with BsmBI and sgRNAs cloned as previously described ${ }^{71}$. H1::sgApc-U6::sgRNA-EFS::mScarlet was generated by Gibson

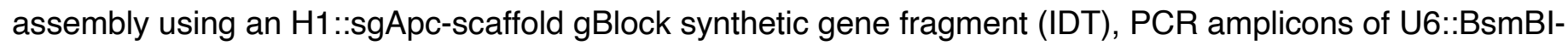
filler-BsmBI-scaffold, EFS promoter, and $m S$ carlet $^{72}$, and the lentiviral backbone from the Trono laboratory (Addgene). This was then digested with BsmBI and a second sgRNA cloned as above. sgRNA sequences: sgApc: 5'-GTCTGCCATCCCTTCACGTT-3'33; sgMsh2: 5'GAACATACATTCGTCAGACCG'; sgMlh1: 5'GAGGGCACCCTGATCACGGTG-3'; sgMsh3: 5'- CTTACTCCGAGCACTCATCG-3'; sgMsh6: 5'CATCAGTGACCGTCTAGATG-3'; non-targeting sgCtl (tdTomato/mScarlet targeting): 5'GGCCACGAGTTCGAGATCGA-3’50; and targeting sgCtl (Olfr102): 5'-GCATCTTTGGCAGTGTCACAG-3', which were used interchangeably with no observed differences in tumorigenesis. PGK::Msh2-EFS::PuroR was generated by Gibson assembly using multiple gBlocks spanning murine Msh2 (C57BL/6), PCR amplicons of PGK and EFS promoters and PuroR, and the Trono lentiviral backbone.

\section{Validation of CRISPR/Cas9 editing and estimation of tumor purity}

To validate efficiency of gene editing by CRISPR/Cas9, 200-250 bp regions spanning sgRNA sites in the genome were amplified and submitted for deep sequencing (CRISPR sequencing) at the Massachusetts General Hospital DNA Core. Tumor purity in colon tumors was estimated by taking the fraction of edited to wild-type alleles at the sgRNA-targeted site in Apc by CRISPR sequencing. Loss of Apc is prerequisite for tumorigenesis in the model, and thus an assumption was made that all tumor cells harbor loss-of-function edits at this locus. Tumor purity in sgMsh2-targeted lung tumors was estimated using WES BAM coverage data spanning exons of the Trp53 flox allele and flanking genes (Wrap53, Atp1b2), which were retrieved using the

bedcov function of SAMtools v1.10. The ratio of median coverage in flanking exons (Wrap53 exons 0-9, Trp53 exon 11, Atp1b2 exons 0-6) versus Trp53 exons flanked by Cre loxP sites (exons 2-10) was calculated in tumors and normal tails. This ratio in tumors, representing extent of Trp53 flox recombination, was then 
normalized to the median ratio across matched normal tails to estimate tumor purity, with the assumption that all tumor cells, but not normal contamination, underwent complete recombination of Trp53flox alleles.

\section{In vitro cell line assays}

Serial live cell imaging of cell lines grown in 96-well plates (Corning) and quantification of confluence was performed with an IncuCyte S3 (Sartorius). Eight replicate wells were seeded with 100 cells each per line and imaged every 3 hours for $\sim 5.5$ days. Murine IFNy (PeproTech) was used for in vitro stimulation of cell lines for 24 hours, followed by live/dead staining (ghost ef780 (Corning), 1:500) in PBS and surface staining in $1 \mathrm{mM}$ EDTA, 25 mM HEPES, 0.5\% heat-inactivated FBS in PBS with anti-H-2K ${ }^{\mathrm{b}}$ (APC, AF6-88.5.5.3, Thermo Fisher), anti-H-2D b (FITC, 28-14-8, Thermo Fisher), and anti-PD-L1 (10F.9G2, PE-Cy7, BioLegend). Samples were run on a BD LSRFortessa using BD FACSDiva v8.0 software. Results were analyzed in FlowJo v10.4.2, excluding dead (ef780 positive) cells.

\section{Phylogenetic tree analysis}

All somatic SNVs and indels called by the WES analysis pipeline in M1-8 SSCs and the 09-2 parental cell line

were considered in constructing a phylogenetic tree. Using the R Bioconductor package 'phangorn' v2.7.0 and a binary presence/absence matrix of mutations across SSCs and 09-2 as input, a tree rooted on 09-2 was constructed. Specifically, the function prachet was used to calculate the tree using the parsimonious ratchet method, and the function acctran was used to calculate branch length.

\section{Statistics and reproducibility}

Statistical analyses and figure generation were performed in R v4.0.2 using built in functions and ggplot2 v3.3.3, beeswarm v0.3.1, corrplot v0.88, eulerr v6.1.0, and RColorBrewer v1.1.2. For statistical assessment of differences in proportionality, Fisher's exact 2x2 test was performed. For continuous data, two-tailed Wilcoxon 
Rank Sum test was performed. Multiple comparison corrections were performed using Holm's method. No statistical method was used to predetermine sample size. In preclinical trials of lung and colon models, only those animals with apparent tumors by $\mu \mathrm{CT}$ or colonoscopy were recruited. No other data were excluded from analyses. Preclinical trials were randomized, and investigators blinded to allocation during dosing, $\mu \mathrm{CT}$ and colonoscopy imaging, and tumor quantification. No experiments presented in this manuscript failed to replicate.

\section{Methods-only references}

45. Johnson, L. et al. Somatic activation of the K-ras oncogene causes early onset lung cancer in mice. Nature 410, 1111-6 (2001).

46. Marino, S., Vooijs, M., van Der Gulden, H., Jonkers, J. \& Berns, A. Induction of medulloblastomas in p53-null mutant mice by somatic inactivation of $\mathrm{Rb}$ in the external granular layer cells of the cerebellum. Genes Dev. 14, 994-1004 (2000).

47. Li, L. et al. Identification of DHODH as a therapeutic target in small cell lung cancer. Sci. Transl. Med. 11, 7852 (2019).

48. Chu, V. T. et al. Efficient generation of Rosa26 knock-in mice using CRISPR/Cas9 in C57BL/6 zygotes. BMC Biotechnol. 16, 4 (2016).

49. Roper, J. et al. Colonoscopy-based colorectal cancer modeling in mice with CRISPR-Cas9 genome editing and organoid transplantation. Nat. Protoc. 13, 217-234 (2018).

50. Sánchez-Rivera, F. J. et al. Rapid modelling of cooperating genetic events in cancer through somatic genome editing. Nature 516, 428-31 (2014).

51. Li, H. Aligning sequence reads, clone sequences and assembly contigs with BWA-MEM. arXiv: Genomics (2013).

52. Van der Auwera, G. A. \& O'Connor, B. D. Genomics in the Cloud: Using Docker, GATK, and WDL in Terra (1st Edition). O’Reilly Media (O’Reilly Media, Inc., 2020).

53. Fan, Y. et al. MuSE: accounting for tumor heterogeneity using a sample-specific error model improves sensitivity and specificity in mutation calling from sequencing data. Genome Biol. 17, 1-11 (2016). 
54. Lai, Z. et al. VarDict: a novel and versatile variant caller for next-generation sequencing in cancer research. Nucleic Acids Res. 44, e108-e108 (2016).

55. Kim, S. et al. Strelka2: fast and accurate calling of germline and somatic variants. Nat. Methods 15, 591-594 (2018).

56. Wang, M. et al. SomaticCombiner: improving the performance of somatic variant calling based on evaluation tests and a consensus approach. Sci. Rep. 10, 1-16 (2020).

57. Kofler, R., Schlötterer, C. \& Lelley, T. SciRoKo: a new tool for whole genome microsatellite search and investigation. Bioinformatics 23, 1683-1685 (2007).

58. Garrison, E. \& Marth, G. Haplotype-based variant detection from short-read sequencing. arXiv: Genomics (2012).

59. Riester, M. et al. PureCN: copy number calling and SNV classification using targeted short read sequencing. Source Code Biol. Med. 11, 1-13 (2016).

60. Li, H. et al. The Sequence Alignment/Map format and SAMtools. Bioinformatics 25, 2078-9 (2009).

61. McLaren, W. et al. The Ensembl variant effect predictor. Genome Biol. 17, 122 (2016).

62. Hundal, J. et al. PVACtools: A computational toolkit to identify and visualize cancer neoantigens. Cancer Immunol. Res. 8, 409-420 (2020).

63. Jurtz, V. et al. NetMHCpan-4.0: Improved peptide-MHC class I interaction predictions integrating eluted ligand and peptide binding affinity data. J. Immunol. 199, 3360-3368 (2017).

64. Andreatta, M. \& Nielsen, M. Gapped sequence alignment using artificial neural networks: Application to the MHC class i system. Bioinformatics 32, 511-517 (2016).

65. Peters, B. \& Sette, A. Generating quantitative models describing the sequence specificity of biological processes with the stabilized matrix method. BMC Bioinformatics 6, (2005).

66. Kim, Y., Sidney, J., Pinilla, C., Sette, A. \& Peters, B. Derivation of an amino acid similarity matrix for peptide:MHC binding and its application as a Bayesian prior. BMC Bioinformatics 10, 394 (2009).

67. Dobin, A. et al. STAR: ultrafast universal RNA-seq aligner. Bioinformatics 29, 15-21 (2013).

68. Picard Tools - By Broad Institute. http://broadinstitute.github.io/picard/.

69. Bankhead, P. et al. QuPath: Open source software for digital pathology image analysis. Sci. Rep. 7, 1-7 
(2017).

70. Tammela, T. et al. A Wnt-producing niche drives proliferative potential and progression in lung adenocarcinoma. Nature 545, 355-359 (2017).

71. Ran, F. A. et al. Genome engineering using the CRISPR-Cas9 system. Nat. Protoc. 8, 2281-2308 (2013).

72. Bindels, D. S. et al. mScarlet: a bright monomeric red fluorescent protein for cellular imaging. Nat. Methods 14, 53-56 (2016).

\section{Acknowledgements}

This work was supported by the NCI Cancer Center Support Grant P30-CA14051, R01 CA233983 and the Howard Hughes Medical Institute. P.M.K.W was supported by a Damon Runyon Fellowship Award. We thank K. Yee, J. Teixeira, K. Anderson, and M. Magendantz for administrative support, and our colleagues in the Jacks laboratory and the broader community at the Koch Institute of MIT for thoughtful discussions and technical advice. We thank the Koch Institute Swanson Biotechnology Center for core support, with particular thanks to K. Cormier and C. Condon for histology support. F.M. and I.C.-C. thank EMBL for funding. We are also grateful for collaboration with T. Westerling and Aiforia in developing automated CNNs for lung tumor grade and IHC quantification.

\section{Author contributions}

P.M.K.W and T.J conceived and directed the study. P.M.K.W, O.S., H.H., N.J.S., A.M.J., W.M.R, and D.A.C. carried out all aspects of the research, animal care and experimentation. F.M. and I.C.-C. designed sequencing analysis pipelines and carried out analysis with P.M.K.W. All other data analysis was carried out by P.M.K.W. Z.A.E. designed and executed the neoantigen prediction pipeline. A.J. provided conceptual and technical guidance in sequencing analysis. S.N. designed dual sgRNA lentiviral constructs. D.Z., M.C.B., A.J., 
J.J.P., and A.M.C produced and validated critical reagents. P.M.K.W, I.C.-C., and T.J. wrote the manuscript, with feedback from all authors.

\section{Competing interest declaration}

T.J. is a member of the Board of Directors of Amgen and Thermo Fisher Scientific, and a co-founder of Dragonfly Therapeutics and T2 Biosystems. T.J. serves on the Scientific Advisory Board of Dragonfly Therapeutics, SQZ Biotech, and Skyhawk Therapeutics. He is the President of Break Through Cancer. None of these affiliations represents a conflict of interest with respect to the design or execution of this study or interpretation of data presented in this manuscript. The Jacks laboratory also currently receives funding from

the Johnson \& Johnson Lung Cancer Initiative and the Lustgarten Foundation for Pancreatic Cancer Research, but this did not support the research described in this manuscript. This work was supported by the Howard Hughes Medical Institute. The remaining authors declare no competing interests. 


\section{Supplementary Files}

This is a list of supplementary files associated with this preprint. Click to download.

- SupplementaryFigureFlowcytometerygatingstrategy.pdf

- Predictedneoantigens500nMIC50andlessWestcottetal9.3.21.txt

- UltradeepseqofSSCdefiningSNVsWestcottetal9.3.21.txt

- WESexonicsomaticvariantsWestcottetal9.3.21.txt 